\title{
Article \\ Analysis and Optimal Control of $\varphi$-Hilfer Fractional Semilinear Equations Involving Nonlocal Impulsive Conditions
}

\author{
Sarra Guechi ${ }^{1}\left(\mathbb{D}\right.$, Rajesh Dhayal $^{2}\left(\mathbb{D}\right.$, Amar Debbouche $^{1, *(\mathbb{D}}$ and Muslim Malik ${ }^{3}(\mathbb{D})$ \\ 1 Department of Mathematics, Guelma University, Guelma 24000, Algeria; guechi.sara@yahoo.fr \\ 2 School of Mathematics, Thapar Institute of Engineering and Technology, Patiala 147 004, India; \\ rajesh.dhayal@thapar.edu \\ 3 School of Basic Sciences, Indian Institute of Technology Mandi, Kamand 175 005, India; \\ muslim@iitmandi.ac.in \\ * Correspondence: amar_debbouche@yahoo.fr
}

check for

updates

Citation: Guechi, S.; Dhayal, R.; Debbouche, A.; Malik, M. Analysis and Optimal Control of $\varphi$-Hilfer Fractional Semilinear Equations Involving Nonlocal Impulsive Conditions. Symmetry 2021, 13, 2084. https://doi.org/10.3390/sym13112084

Academic Editors: Francisco

Martínez González and Mohammed K. A. Kaabar

Received: 1 August 2021

Accepted: 30 October 2021

Published: 3 November 2021

Publisher's Note: MDPI stays neutral with regard to jurisdictional claims in published maps and institutional affiliations.

Copyright: (c) 2021 by the authors. Licensee MDPI, Basel, Switzerland. This article is an open access article distributed under the terms and conditions of the Creative Commons Attribution (CC BY) license (https:// creativecommons.org/licenses/by/ $4.0 /)$.

\begin{abstract}
The goal of this paper is to consider a new class of $\varphi$-Hilfer fractional differential equations with impulses and nonlocal conditions. By using fractional calculus, semigroup theory, and with the help of the fixed point theorem, the existence and uniqueness of mild solutions are obtained for the proposed fractional system. Symmetrically, we discuss the existence of optimal controls for the $\varphi$-Hilfer fractional control system. Our main results are well supported by an illustrative example.
\end{abstract}

Keywords: $\varphi$-Hilfer fractional system with impulses; semigroup theory; nonlocal conditions; optimal controls

\section{Introduction}

In recent years, a lot of research attention has been paid to the study of fractional calculus, which is considered as a generalization of classical derivatives and integrals to non-integer order. Phenomena with memory and hereditary characteristics that arise in ecology, biology, medicine, electrical engineering, and mechanics, etc, may be well modelled by using fractional differential equations (FDEs for short). For more details on FDEs and its applications, see [1-5] and the references therein. In [6], Hilfer derived a new two-parameter fractional derivative $\mathrm{D}_{a^{+}}^{\sigma_{1}, \sigma_{2}}$ of order $\sigma_{1}$ and type $\sigma_{2}$, which is called Hilfer fractional derivative that combines the Riemann-Liouville and Caputo fractional derivatives. This kind of parameter produces more types of stationary states and gives an extra degree of freedom on the initial conditions. Systems based on Hilfer fractional derivatives are considered by many authors, see [7-11] and the references therein. Recently, Sousa and Oliveira [12] introduced a new fractional derivative with respect to another $\varphi$-function the so-called $\varphi$-Hilfer fractional derivative, and discussed their properties as well as important results of the fractional calculus. For more recent works on $\varphi$-Hilfer fractional derivative and its applications, we refer to [13-17] and the references therein.

Many real-world phenomena and processes which are subjected to external influences for a small time interval during their evolution can be represented as an impulsive differential equations. The impulsive differential equations have become the natural framework for modelling of many evolving processes and phenomena studied in the field of science and engineering such as in mechanical systems, biological systems, population dynamics, physics, economy, and control theory. Recently, based on the theory of semigroup and fixed point approach, many authors studied the qualitative properties of solutions for impulsive differential equations of order one and non-integer [18-24] and the references therein. The optimal control problem (OCP for short) plays a crucial role in biomedicine, for example, model cancer chemotherapy and recently applied to epidemiological models. When FDEs describe the system dynamics and the cost functional, an OCP reduces to a fractional optimal control problem. The fractional OCP refers to optimize the cost functional subject to dynamical constraints on the control parameter and state variables that 
having fractional models. For more recent works on OCP, see [25-30] and the references therein. Harrat et al. [31] investigated the existence of optimal controls for Hilfer fractional impulsive evolution inclusions with Clarke subdifferential. Moreover, optimal control problems for $\varphi$-Hilfer fractional impulsive differential equations are rarely available in the literature which serves as a motivation to our research work in this paper.

Motivated by the above facts, we consider following $\varphi$-Hilfer fractional impulsive differential system:

$$
\left\{\begin{array}{l}
{ }^{H} \mathrm{D}_{t_{\gamma}}^{\sigma_{1}, \sigma_{2}: \varphi} z(t)=\mathcal{A} z(t)+\Delta(t, z(t)), t \in(0, b]-\left\{t_{1}, t_{2}, \ldots, t_{\mathcal{H}}\right\}, \\
I_{t_{\gamma}^{+}}^{\left(1-\sigma_{1}\right)\left(1-\sigma_{2}\right) ; \varphi} z\left(t_{\gamma}^{+}\right)=z\left(t_{\gamma}^{-}\right)+\mathcal{I}_{\gamma}\left(z\left(t_{\gamma}^{-}\right)\right), \gamma=1,2, \ldots, \mathcal{H}, \\
I_{0^{+}}^{\left(1-\sigma_{1}\right)\left(1-\sigma_{2}\right) ; \varphi}[z(t)]_{t=0}+\mathcal{G}(z)=z_{0},
\end{array}\right.
$$

where ${ }^{H} \mathrm{D}_{t_{\gamma^{+}}{ }^{+}}^{\sigma_{1}, \sigma_{2} ; \varphi}$ denotes the $\varphi$-Hilfer fractional derivative of order $1 / 2<\sigma_{1}<1,0<\sigma_{2}<1$ and the state $z(\cdot)$ takes values in a Hilbert space $E$ and $\mathcal{J}_{0}=[0, b], 0=t_{0}<t_{1}<\cdots<$ $t_{\mathcal{H}}<t_{\mathcal{H}+1}=b . \mathcal{A}$ is the generator of a $C_{0}$-semigroup $\{\mathcal{T}(t)\}_{t \geq 0}$ on $E$. As usual $z\left(t_{\gamma}^{+}\right)$and $z\left(t_{\gamma}^{-}\right)$are the right and left limits of $z$ at the point $t_{\gamma}$, respectively. $\mathcal{I}_{\gamma}: E \rightarrow E$ are impulsive functions that characterize the jump of $z$ at points $t_{\gamma}$. The functions $\Delta: \mathcal{J}_{0} \times E \rightarrow E$, $\mathcal{G}: C\left(\mathcal{J}_{0}, E\right) \rightarrow E$ are some suitable functions that will be specified later.

The rest of the manuscript is organized as follows. In Section 2, we recall some important concepts and results. In Sections 3 and 4, we derived the mild solution by using semigroup as well as probability density function and proved the existence of mild solutions for the proposed fractional system, receptively. In Section 5, we investigated the existence of optimal controls for the $\varphi$-Hilfer fractional control system. Moreover, in Section 6, an example is presented to demonstrate the applicability of the obtained symmetry results.

\section{Preliminaries}

Let $\mathcal{J}_{1}=[a, b]$ and $\varphi \in C^{m}\left(\mathcal{J}_{1}, \mathbb{R}\right)$ an increasing function such that $\varphi^{\prime}(t) \neq 0, \forall t \in \mathcal{J}_{1}$.

Definition 1. The $\varphi$-Riemann fractional integral of order $\sigma_{1}>0$ of the function $\mathcal{R}$ is given by

$$
I_{a^{+}}^{\sigma_{1} ; \varphi} \mathcal{R}(t)=\frac{1}{\Gamma\left(\sigma_{1}\right)} \int_{a}^{t}(\varphi(t)-\varphi(s))^{\sigma_{1}-1} \mathcal{R}(s) \varphi^{\prime}(s) d s .
$$

Definition 2. The $\varphi$-Riemann-Liouville fractional derivative of function $\mathcal{R}$ of order $\sigma_{1}(m-1<$ $\left.\sigma_{1}<m, m \in \mathbb{N}\right)$, is defined by

$$
\mathrm{D}_{a^{+}}^{\sigma_{1} ; \varphi} \mathcal{R}(t)=\left(\frac{1}{\varphi^{\prime}(t)} \frac{d}{d t}\right)^{m} I_{a^{+}}^{m-\sigma_{1} ; \varphi} \mathcal{R}(t)=\frac{\left(\frac{1}{\varphi^{\prime}(t)} \frac{d}{d t}\right)^{m}}{\Gamma\left(m-\sigma_{1}\right)} \int_{a}^{t}(\varphi(t)-\varphi(s))^{m-\sigma_{1}-1} \mathcal{R}(s) d s,
$$

where $m=\left[\sigma_{1}\right]+1$.

Definition 3. The $\varphi$-Hilfer fractional derivative of function $\mathcal{R}$ of order $\sigma_{1}\left(m-1<\sigma_{1}<m\right.$, $m \in \mathbb{N}$ ) and type $0 \leq \sigma_{2} \leq 1$, is defined by

$$
{ }^{H} \mathrm{D}_{a^{+}}^{\sigma_{1}, \sigma_{2} ; \varphi} \mathcal{R}(t)=I_{a^{+}}^{\sigma_{2}\left(m-\sigma_{1}\right) ; \varphi}\left(\frac{1}{\varphi^{\prime}(t)} \frac{d}{d t}\right)^{m} I_{a+}^{\left(1-\sigma_{2}\right)\left(m-\sigma_{1}\right) ; \varphi} \mathcal{R}(t) .
$$

The $\varphi$-Hilfer fractional derivative can be written as

$$
{ }^{H} \mathrm{D}_{a^{+}}^{\sigma_{1}, \sigma_{2} ; \varphi} \mathcal{R}(t)=I_{a^{+}}^{\delta-\sigma_{1} ; \varphi} \mathrm{D}_{a^{+}}^{\delta ; \varphi} \mathcal{R}(t),
$$

with $\delta=\left(\sigma_{1}+\sigma_{2}\left(m-\sigma_{1}\right)\right)$. 
Lemma 1 ([12]). If $\mathcal{R} \in \mathcal{C}^{m}[a, b], m-1<\sigma_{1}<m$ and $0 \leq \sigma_{2} \leq 1$, then

$$
I_{a+}^{\sigma_{1} ; \varphi}{ }^{H} \mathrm{D}_{a^{+}}^{\sigma_{1}, \sigma_{2} ; \varphi} \mathcal{R}(t)=\mathcal{R}(t)-\sum_{k=1}^{m} \frac{(\varphi(t)-\varphi(a))^{\delta-k}}{\Gamma(\delta-k+1)} \mathcal{R}_{\varphi}^{[m-k]} I_{a+}^{\left(1-\sigma_{2}\right)\left(m-\sigma_{1}\right) ; \varphi} \mathcal{R}(a) .
$$

Lemma 2 ([12]). Let $\sigma_{1}>0$ and $\sigma_{2}>0$, then $I_{a^{+}}^{\sigma_{1}, \varphi}(\varphi(t)-\varphi(a))^{\sigma_{2}-1}=\frac{\Gamma\left(\sigma_{2}\right)}{\Gamma\left(\sigma_{2}+\sigma_{1}\right)}(\varphi(t)-$ $\varphi(a))^{\sigma_{2}+\sigma_{1}-1}$.

Definition 4. Let $z, \varphi:[c, \infty) \rightarrow \mathbb{R}$ be the functions such that $\varphi(t)$ is continuous and $\varphi^{\prime}(t)>0$ on $[0, \infty)$. Then the generalized Laplace transform of function $z(t)$ is given by

$$
\mathcal{L}_{\varphi}\{z(t)\}(s)=\int_{\mathcal{C}}^{\infty} e^{-s(\varphi(t)-\varphi(a))} z(t) \varphi^{\prime}(t) d t, \text { for all } s .
$$

For comprehensive details on $\varphi$-Hilfer fractional derivative and its properties, we refer to papers $[12,14,17]$.

Consider the weighted space [14] defined as

$$
\mathcal{C}_{1-\rho ; \varphi}\left(\mathcal{J}_{0}, E\right)=\left\{z:[0, b] \rightarrow E:\left(\varphi(t)-\varphi\left(t_{\gamma}\right)\right)^{1-\rho} z(t) \in C\left(\mathcal{J}_{0}, E\right)\right\} .
$$

Define the space of piecewise continuous functions as

$$
\begin{aligned}
\mathcal{P C}_{1-\rho ; \varphi}\left(\mathcal{J}_{0}, E\right)= & \left\{z:[0, b] \rightarrow E: z \in \mathcal{C}_{1-\rho ; \varphi}\left(\left(t_{\gamma}, t_{\gamma+1}\right], E\right), \gamma=1,2, \ldots, \mathcal{H}, I_{t_{\gamma}}^{(1-\rho) ; \varphi} z\left(t_{\gamma}^{+}\right)\right. \\
& \text {and } \left.I_{t_{\gamma}}^{(1-\rho) ; \varphi} z\left(t_{\gamma}^{-}\right)=I_{t_{\gamma}}^{(1-\rho) ; \varphi} z\left(t_{\gamma}\right) \text { exists for } \gamma=1,2, \ldots, \mathcal{H}, \rho=\sigma_{1}+\sigma_{2}-\sigma_{2} \sigma_{1}\right\}
\end{aligned}
$$

Clearly, $\mathcal{P C}(E)=\mathcal{P C}_{1-\rho ; \varphi}\left(\mathcal{J}_{0}, E\right)$ is a Banach space with the norm

$$
\|z\|_{\mathcal{P C}}=\max _{\gamma=1,2, \ldots, \mathcal{H}}\left\{\sup _{t \in\left(t_{\gamma}, t_{\gamma+1}\right]}\left\|\left[\varphi(t)-\varphi\left(t_{\gamma}\right)\right]^{1-\rho} z(t)\right\|\right\} .
$$

\section{Representation of Mild Solution}

Lemma 3. To reduce the generalized form (1), we consider the linear $\varphi$-Hilfer fractional differential system:

$$
\left\{\begin{array}{l}
{ }^{H} \mathrm{D}_{0^{+}}^{\sigma_{1}, \sigma_{2}: \varphi} z(t)=\mathcal{A} z(t)+\Delta(t), t \in(0, b] \\
I_{0^{+}}^{\left(1-\sigma_{1}\right)\left(1-\sigma_{2}\right) ; \varphi}[z(t)]_{t=0}=z_{0}
\end{array}\right.
$$

has a mild solution, which is defined as

$$
z(t)=\mathcal{S}_{\varphi}^{\sigma_{1}, \sigma_{2}}(t, 0) z_{0}+\int_{0}^{t}(\varphi(t)-\varphi(s))^{\sigma_{1}-1} \mathcal{T}_{\varphi}^{\sigma_{1}}(t, s) \Delta(s) \varphi^{\prime}(s) d s,
$$

where

$$
\begin{aligned}
\mathcal{P}_{\varphi}^{\sigma_{1}}(t, s) z & =\int_{0}^{\infty} \phi_{\sigma_{1}}(\theta) \mathcal{T}\left((\varphi(t)-\varphi(s))^{\sigma_{1}} \theta\right) z d \theta, \\
\mathcal{S}_{\varphi}^{\sigma_{1}, \sigma_{2}}(t, s) z & =I_{a^{+}}^{\left(1-\sigma_{1}\right)\left(\sigma_{2}-1\right) ; \varphi} \mathcal{P}_{\varphi}^{\sigma_{1}}(t, s) z, \\
\mathcal{T}_{\varphi}^{\sigma_{1}}(t, s) z & =\sigma_{1} \int_{0}^{\infty} \theta \phi_{\sigma_{1}}(\theta) \mathcal{T}\left((\varphi(t)-\varphi(s))^{\sigma_{1}} \theta\right) z d \theta, 0 \leq s \leq t \leq b,
\end{aligned}
$$

with

$$
\phi_{\sigma_{1}}(\theta) \geq 0 \text { for } \theta \geq 0, \quad \int_{0}^{\infty} \phi_{\sigma_{1}}(\theta) d \theta=1, \quad \text { and } \quad \int_{0}^{\infty} \theta \phi_{\sigma_{1}}(\theta) d \theta=\frac{1}{\Gamma\left(1+\sigma_{1}\right)} .
$$

Proof. Rewrite the problem (2) in the equivalent integral equation 


$$
z(t)=\frac{(\varphi(t)-\varphi(0))^{\left(1-\sigma_{1}\right)\left(\sigma_{2}-1\right)}}{\Gamma\left(\sigma_{2}\left(1-\sigma_{1}\right)+\sigma_{1}\right)} z_{0}+\frac{1}{\Gamma\left(\sigma_{1}\right)} \int_{0}^{t}(\varphi(t)-\varphi(s))^{\sigma_{1}-1}[\mathcal{A} z(s)+\Delta(s)] \varphi^{\prime}(s) d s,
$$

provided that the integral in Equation (4) exists. Let $\beta>0$. Applying the generalized Laplace transform

$$
Z(\beta)=\frac{1}{\beta^{\sigma_{2}\left(1-\sigma_{1}\right)+\sigma_{1}}} z_{0}+\frac{1}{\beta^{\sigma_{1}}}(\mathcal{A} Z(\beta)+\hat{\Delta}(\beta)),
$$

where

$$
\begin{aligned}
& Z(\beta)=\int_{0}^{\infty} e^{-\beta(\varphi(\mu)-\varphi(0))} z(\mu) \varphi^{\prime}(\mu) d \mu, \\
& \hat{\Delta}(\beta)=\int_{0}^{\infty} e^{-\beta(\varphi(\mu)-\varphi(0))} \Delta(\mu) \varphi^{\prime}(\mu) d \mu .
\end{aligned}
$$

It follows that

$$
\begin{aligned}
Z(\beta) & =\beta^{\sigma_{2}\left(\sigma_{1}-1\right)}\left(\beta^{\sigma_{1}} I-\mathcal{A}\right)^{-1} z_{0}+\left(\beta^{\sigma_{1}} I-\mathcal{A}\right)^{-1} \hat{\Delta}(\beta) \\
& =\beta^{\sigma_{2}\left(\sigma_{1}-1\right)} \int_{0}^{\infty} e^{-\beta^{\sigma_{1}} s} \mathcal{T}(s) z_{0} d s+\int_{0}^{\infty} e^{-\beta^{\sigma_{1}} s} \mathcal{T}(s) \hat{\Delta}(\beta) d s
\end{aligned}
$$

Taking $s=\hat{t}^{\sigma_{1}}$, we obtain

$$
\begin{aligned}
Z(\beta)= & \sigma_{1} \beta^{\left(\sigma_{1}-1\right)\left(\sigma_{2}-1\right)} \int_{0}^{\infty}(\beta \hat{t})^{\sigma_{1}-1} e^{-(\beta \hat{t})^{\sigma_{1}}} \mathcal{T}\left(\hat{t}^{\sigma_{1}}\right) z_{0} d \hat{t}+\sigma_{1} \int_{0}^{\infty} \hat{t}^{\sigma_{1}-1} e^{-(\beta \hat{t})^{\sigma_{1}}} \mathcal{T}\left(\hat{t}^{\sigma_{1}}\right) \hat{\Delta}(\beta) d \hat{t} \\
= & \beta^{\left(\sigma_{1}-1\right)\left(\sigma_{2}-1\right)} I_{1}+I_{2}, \\
& \text { where }
\end{aligned}
$$

$$
\begin{aligned}
& I_{1}=\sigma_{1} \int_{0}^{\infty}(\beta \hat{t})^{\sigma_{1}-1} e^{-(\beta \hat{t})^{\sigma_{1}}} \mathcal{T}\left(\hat{t}^{\sigma_{1}}\right) z_{0} d \hat{t}, \\
& I_{2}=\sigma_{1} \int_{0}^{\infty} \hat{t}^{\sigma_{1}-1} e^{-(\beta \hat{t})^{\sigma_{1}}} \mathcal{T}\left(\hat{t}^{\sigma_{1}}\right) \hat{\Delta}(\beta) d \hat{t} .
\end{aligned}
$$

Taking $\hat{t}=\varphi(t)-\varphi(0)$, we obtain

$$
\begin{aligned}
I_{1}= & \sigma_{1} \int_{0}^{\infty} \beta^{\sigma_{1}-1}(\varphi(t)-\varphi(0))^{\sigma_{1}-1} e^{-(\beta(\varphi(t)-\varphi(0)))^{\sigma_{1}}} \mathcal{T}\left((\varphi(t)-\varphi(0))^{\sigma_{1}}\right) z_{0} \varphi^{\prime}(t) d t \\
= & \int_{0}^{\infty} \frac{-1}{\beta} \frac{d}{d t}\left(e^{-(\beta(\varphi(t)-\varphi(0)))^{\sigma_{1}}}\right) \mathcal{T}\left((\varphi(t)-\varphi(0))^{\sigma_{1}}\right) z_{0} d t . \\
I_{2}= & \sigma_{1} \int_{0}^{\infty}(\varphi(t)-\varphi(0))^{\sigma_{1}-1} e^{-(\beta(\varphi(t)-\varphi(0)))^{\sigma_{1}}} \mathcal{T}\left((\varphi(t)-\varphi(0))^{\sigma_{1}}\right) \hat{\Delta}(\beta) \varphi^{\prime}(t) d t \\
= & \int_{0}^{\infty} \int_{0}^{\infty} \sigma_{1}(\varphi(t)-\varphi(0))^{\sigma_{1}-1} e^{-(\beta(\varphi(t)-\varphi(0)))^{\sigma_{1}}} \mathcal{T}\left((\varphi(t)-\varphi(0))^{\sigma_{1}}\right) \\
& \times e^{-(\beta(\varphi(s)-\varphi(0)))} \Delta(s) \varphi^{\prime}(s) \varphi^{\prime}(t) d s d t .
\end{aligned}
$$

We consider the following one-sided stable probability density

$$
\rho_{\sigma_{1}}(\theta)=\frac{1}{\pi} \sum_{k=1}^{\infty}(-1)^{k-1} \theta^{-\sigma_{1} k-1} \frac{\Gamma\left(\sigma_{1} k+1\right)}{k !} \sin \left(k \pi \sigma_{1}\right), \theta \in(0, \infty),
$$


whose integration is given by

$$
\int_{0}^{\infty} e^{-\beta \theta} \rho_{\sigma_{1}}(\theta) d \theta=e^{-\beta^{\sigma_{1}}}, \sigma_{1} \in(0,1) .
$$

Using Equation (5), we obtain

$$
\begin{aligned}
I_{1} & =\int_{0}^{\infty} \frac{-1}{\beta} \frac{d}{d t}\left(\int_{0}^{\infty} e^{-(\beta(\varphi(t)-\varphi(0))) \theta} \rho_{\sigma_{1}}(\theta) d \theta\right) \mathcal{T}\left((\varphi(t)-\varphi(0))^{\sigma_{1}}\right) z_{0} d t \\
& =\int_{0}^{\infty} \int_{0}^{\infty} \theta \rho_{\sigma_{1}}(\theta) e^{-(\beta(\varphi(t)-\varphi(0))) \theta} \mathcal{T}\left((\varphi(t)-\varphi(0))^{\sigma_{1}}\right) z_{0} \varphi^{\prime}(t) d \theta d t \\
& =\int_{0}^{\infty} e^{-(\beta(\varphi(t)-\varphi(0)))}\left(\int_{0}^{\infty} \rho_{\sigma_{1}}(\theta) \mathcal{T}\left(\frac{(\varphi(t)-\varphi(0))^{\sigma_{1}}}{\theta^{\sigma_{1}}}\right) d \theta\right) z_{0} \varphi^{\prime}(t) d t .
\end{aligned}
$$

and

$$
\begin{aligned}
& I_{2}=\int_{0}^{\infty} \int_{0}^{\infty} \int_{0}^{\infty} \sigma_{1}(\varphi(t)-\varphi(0))^{\sigma_{1}-1} \rho_{\sigma_{1}}(\theta) e^{-(\beta(\varphi(t)-\varphi(0))) \theta} \mathcal{T}\left((\varphi(t)-\varphi(0))^{\sigma_{1}}\right) e^{-(\beta(\varphi(s)-\varphi(0)))} \\
& \times \Delta(s) \varphi^{\prime}(s) \varphi^{\prime}(t) d \theta d s d t \\
& =\int_{0}^{\infty} \int_{0}^{\infty} \int_{0}^{\infty} \sigma_{1} e^{-(\beta(\varphi(t)+\varphi(s)-2 \varphi(0)))} \frac{(\varphi(t)-\varphi(0))^{\sigma_{1}-1}}{\theta^{\sigma_{1}}} \rho_{\sigma_{1}}(\theta) \mathcal{T}\left(\frac{(\varphi(t)-\varphi(0))^{\sigma_{1}}}{\theta^{\sigma_{1}}}\right) \\
& \times \Delta(s) \varphi^{\prime}(s) \varphi^{\prime}(t) d \theta d s d t \\
& =\int_{0}^{\infty} \int_{0}^{\mu} \int_{0}^{\infty} \sigma_{1} e^{-(\beta(\varphi(\mu)-\varphi(0)))} \rho_{\sigma_{1}}(\theta) \frac{(\varphi(t)-\varphi(0))^{\sigma_{1}-1}}{\theta^{\sigma_{1}}} \mathcal{T}\left(\frac{(\varphi(t)-\varphi(0))^{\sigma_{1}}}{\theta^{\sigma_{1}}}\right) \\
& \left.\times \Delta\left(\varphi^{-1}(\varphi(\mu)-\varphi(t)+\varphi(0))\right)\right) \varphi^{\prime}(\mu) \varphi^{\prime}(t) d \theta d t d \mu \\
& =\int_{0}^{\infty} e^{-(\beta(\varphi(\mu)-\varphi(0)))}\left(\int_{0}^{\mu} \int_{0}^{\infty} \sigma_{1} \rho_{\sigma_{1}}(\theta) \frac{(\varphi(\mu)-\varphi(s))^{\sigma_{1}-1}}{\theta^{\sigma_{1}}} \mathcal{T}\left(\frac{(\varphi(\mu)-\varphi(s))^{\sigma_{1}}}{\theta^{\sigma_{1}}}\right)\right. \\
& \left.\times \Delta(s) \varphi^{\prime}(s) d \theta d s\right) \varphi^{\prime}(\mu) d \mu .
\end{aligned}
$$

\section{Hence, we obtain}

$$
\begin{aligned}
Z(\beta)= & \beta^{\left(\sigma_{1}-1\right)\left(\sigma_{2}-1\right)} \int_{0}^{\infty} e^{-(\beta(\varphi(t)-\varphi(0)))}\left(\int_{0}^{\infty} \rho_{\sigma_{1}}(\theta) \mathcal{T}\left(\frac{\varphi(t)-\varphi(0))^{\sigma_{1}}}{\theta^{\sigma_{1}}}\right) z_{0} d \theta\right) \varphi^{\prime}(t) d t \\
+ & \int_{0}^{\infty} e^{-(\beta(\varphi(\mu)-\varphi(0)))}\left(\int_{0}^{\mu} \int_{0}^{\infty} \sigma_{1} \rho_{\sigma_{1}}(\theta) \frac{(\varphi(\mu)-\varphi(s))^{\sigma_{1}-1}}{\theta^{\sigma_{1}}} \mathcal{T}\left(\frac{(\varphi(\mu)-\varphi(s))^{\sigma_{1}}}{\theta^{\sigma_{1}}}\right)\right. \\
& \left.\times \Delta(s) \varphi^{\prime}(s) d \theta d s\right) \varphi^{\prime}(\mu) d \mu .
\end{aligned}
$$

By using inverse Laplace transform, we obtain

$$
\begin{aligned}
z(t) & =I_{a^{+}}^{\left(1-\sigma_{1}\right)\left(\sigma_{2}-1\right) ; \varphi} \int_{0}^{\infty} \rho_{\sigma_{1}}(\theta) \mathcal{T}\left(\frac{\varphi(t)-\varphi(0))^{\sigma_{1}}}{\theta^{\sigma_{1}}}\right) z_{0} d \theta \\
& +\int_{0}^{t} \int_{0}^{\infty} \sigma_{1} \rho_{\sigma_{1}}(\theta) \frac{(\varphi(t)-\varphi(s))^{\sigma_{1}-1}}{\theta^{\sigma_{1}}} \mathcal{T}\left(\frac{(\varphi(t)-\varphi(s))^{\sigma_{1}}}{\theta^{\sigma_{1}}}\right) \Delta(s) \varphi^{\prime}(s) d \theta d s .
\end{aligned}
$$

Thus, we obtain

$$
\begin{aligned}
z(t) & \left.=I_{a^{+}}^{\left(1-\sigma_{1}\right)\left(\sigma_{2}-1\right) ; \varphi} \int_{0}^{\infty} \phi_{\sigma_{1}}(\theta) \mathcal{T}(\varphi(t)-\varphi(0))^{\sigma_{1}} \theta\right) z_{0} d \theta \\
& +\sigma_{1} \int_{0}^{t} \int_{0}^{\infty} \theta \phi_{\sigma_{1}}(\theta)(\varphi(t)-\varphi(s))^{\sigma_{1}-1} \mathcal{T}\left((\varphi(t)-\varphi(s))^{\sigma_{1}} \theta\right) \Delta(s) \varphi^{\prime}(s) d \theta d s,
\end{aligned}
$$


where $\phi_{\sigma_{1}}(\theta)=\frac{1}{\sigma_{1}} \theta^{-1-\frac{1}{\sigma_{1}}} \rho_{\sigma_{1}}\left(\theta^{-\frac{1}{\sigma_{1}}}\right)$ is the probability density function defined on $(0, \infty)$. For any $z \in E$, the operators $\mathcal{S}_{\varphi}^{\sigma_{1} \sigma_{2}}(t, s)$ and $\mathcal{T}_{\varphi}^{\sigma_{1}}(t, s)$ defined as

$$
\begin{aligned}
\mathcal{P}_{\varphi}^{\sigma_{1}}(t, s) z & =\int_{0}^{\infty} \phi_{\sigma_{1}}(\theta) \mathcal{T}\left((\varphi(t)-\varphi(s))^{\sigma_{1}} \theta\right) z d \theta \\
\mathcal{S}_{\varphi}^{\sigma_{1}, \sigma_{2}}(t, s) z & =I_{a^{+}}^{\left(1-\sigma_{1}\right)\left(\sigma_{2}-1\right) ; \varphi} \mathcal{P}_{\varphi}^{\sigma_{1}}(t, s) z
\end{aligned}
$$

and

$$
\mathcal{T}_{\varphi}^{\sigma_{1}}(t, s) z=\sigma_{1} \int_{0}^{\infty} \theta \phi_{\sigma_{1}}(\theta) \mathcal{T}\left((\varphi(t)-\varphi(s))^{\sigma_{1}} \theta\right) z d \theta, 0 \leq s \leq t \leq b .
$$

Hence, we obtain

$$
z(t)=\mathcal{S}_{\varphi}^{\sigma_{1}, \sigma_{2}}(t, 0) z_{0}+\int_{0}^{t}(\varphi(t)-\varphi(s))^{\sigma_{1}-1} \mathcal{T}_{\varphi}^{\sigma_{1}}(t, s) \Delta(s) \varphi^{\prime}(s) d s .
$$

Remark 1. Let $\mathcal{A}$ be the generator of a $C_{0}$-semigroup $\{\mathcal{T}(t)\}_{t \geq 0}$ on $E$. Then there exists $\mathcal{M} \geq 1$ such that $\mathcal{M}=\sup _{t \in[0, b]} \mathcal{T}(t)$

Lemma 4 ([17,32]). The operators $\mathcal{S}_{\varphi}^{\sigma_{1}, \sigma_{2}}$ and $\mathcal{T}_{\varphi}^{\sigma_{1}}$ have the subsequent conditions

1. $\mathcal{S}_{\varphi}^{\sigma_{1}, \sigma_{2}}(t, s)$ and $\mathcal{T}_{\varphi}^{\sigma_{1}}(t, s)$ are linear and bounded operators for any fixed $t \geq s \geq 0$, and

$$
\begin{aligned}
\left\|\mathcal{S}_{\varphi}^{\sigma_{1}, \sigma_{2}}(t, s)(z)\right\| & \leq \frac{\mathcal{M}(\varphi(b)-\varphi(0))^{\left(1-\sigma_{1}\right)\left(\sigma_{2}-1\right)}}{\Gamma\left(\sigma_{1}+\sigma_{2}-\sigma_{1} \sigma_{2}\right)}\|z\|=\mathcal{M}_{1}\|z\|, \\
\left\|\mathcal{T}_{\varphi}^{\sigma_{1}}(t, s)(z)\right\| & \leq \frac{\sigma_{1} \mathcal{M}}{\Gamma\left(1+\sigma_{1}\right)}\|z\|=\frac{\mathcal{M}}{\Gamma\left(\sigma_{1}\right)}\|z\|=\mathcal{M}_{2}\|z\| .
\end{aligned}
$$

2. If $\mathcal{T}(t)$ is compact operator for all $t>0$, then $\mathcal{S}_{\varphi}^{\sigma_{1}, \sigma_{2}}(t, s), \mathcal{T}_{\varphi}^{\sigma_{1}}(t, s)$ are compact for all $t, s>0$. Hence, $\mathcal{S}_{\varphi}^{\sigma_{1}, \sigma_{2}}(t, s)$ and $\mathcal{T}_{\varphi}^{\sigma_{1}}(t, s)$ are strongly continuous.

3. The operators $\mathcal{S}_{\varphi}^{\sigma_{1}, \sigma_{2}}(t, s)$ and $\mathcal{T}_{\varphi}^{\sigma_{1}}(t, s)$ are strongly continuous. For every $z \in E$ and $0 \leq s \leq t_{1}<t_{2} \leq b$, we have

$$
\left\|\mathcal{S}_{\varphi}^{\sigma_{1}, \sigma_{2}}\left(t_{2}, s\right) z-\mathcal{S}_{\varphi}^{\sigma_{1}, \sigma_{2}}\left(t_{1}, s\right) z\right\| \rightarrow 0 \text { and }\left\|\mathcal{T}_{\varphi}^{\sigma_{1}}\left(t_{2}, s\right) z-\mathcal{T}_{\varphi}^{\sigma_{1}}\left(t_{1}, s\right) z\right\| \rightarrow 0 \text { as } t_{1} \rightarrow t_{2} .
$$

Definition 5. A function $z \in \mathcal{P C}(E)$ is called a mild solution of problem (1) if for every $t \in \mathcal{J}_{0}, z(t)$ fulfills $I_{0^{+}}^{\left(1-\sigma_{1}\right)\left(1-\sigma_{2}\right) ; \varphi}[z(t)]_{t=0}+\mathcal{G}(z)=z_{0}, I_{t_{\gamma}}^{\left(1-\sigma_{1}\right)\left(1-\sigma_{2}\right) ; \varphi} z\left(t_{\gamma}^{+}\right)=z\left(t_{\gamma}^{-}\right)+$ $\mathcal{I}_{\gamma}\left(z\left(t_{\gamma}^{-}\right)\right), \gamma=1,2, \ldots, \mathcal{H}$, and

$$
z(t)=\mathcal{S}_{\varphi}^{\sigma_{1}, \sigma_{2}}(t, 0)\left[z_{0}-\mathcal{G}(z)\right]+\int_{0}^{t}(\varphi(t)-\varphi(s))^{\sigma_{1}-1} \mathcal{T}_{\varphi}^{\sigma_{1}}(t, s) \Delta(s, z(s)) \varphi^{\prime}(s) d s,
$$

for every $t \in\left[0, t_{1}\right]$ and

$z(t)=\mathcal{S}_{\varphi}^{\sigma_{1}, \sigma_{2}}\left(t, t_{\gamma}\right)\left[z\left(t_{\gamma}^{-}\right)+\mathcal{I}_{\gamma}\left(z\left(t_{\gamma}^{-}\right)\right)\right]+\int_{t_{\gamma}}^{t}(\varphi(t)-\varphi(s))^{\sigma_{1}-1} \mathcal{T}_{\varphi}^{\sigma_{1}}(t, s) \Delta(s, z(s)) \varphi^{\prime}(s) d s$,

for every $t \in\left(t_{\gamma}, t_{\gamma+1}\right]$.

\section{Existence and Uniqueness}

In this section, we prove the existence outcomes of the proposed system (1). Let us assume the following hypotheses

[X1]: $\mathcal{T}(t)$ is compact for every $t>0$.

[X2]: The function $\Delta: \mathcal{J}_{0} \times E \rightarrow E$ satisfies 
(a) For all $z \in E$, the function $t \rightarrow \Delta(t, z)$ is strongly measurable and the function $\Delta(t, \cdot): E \rightarrow E$ is continuous for a.e $t \in \mathcal{J}_{0}$.

(b) There exists a continuous function $\hat{\mathcal{K}}_{\Delta} \in L^{1}\left(\mathcal{J}_{0}, \mathbb{R}^{+}\right)$such that

$$
\|\Delta(t, z)\| \leq \hat{\mathcal{K}}_{\Delta}(t)\|z\|, \forall(t, z) \in \mathcal{J}_{0} \times E,
$$

with $\mathcal{K}_{\Delta}=\sup _{t \in \mathcal{J}_{0}} \hat{\mathcal{K}}_{\Delta}(t)$.

[X3]: The function $\mathcal{G}: C\left(\mathcal{J}_{0}, E\right) \rightarrow E$ is Lipschitz continuous, i.e.; there exists a positive constant $\hat{\mathcal{K}}_{\mathcal{G}}$ such that

$$
\left\|\mathcal{G}\left(z_{1}\right)-\mathcal{G}\left(z_{2}\right)\right\| \leq \hat{\mathcal{K}}_{\mathcal{G}}\left\|z_{1}-z_{2}\right\|, \forall z_{1}, z_{2} \in E
$$

[X4]: For every $z, z_{1}, z_{2} \in E$ and all $t \in\left(t_{\gamma}, t_{\gamma+1}\right], \gamma=1,2, \ldots, \mathcal{H}$, there exist $\mathcal{D}_{\gamma}, \mathcal{K}_{\gamma}>0$, satisfies

$$
\left\|\mathcal{I}_{\gamma}\left(z\left(t_{\gamma}^{-}\right)\right)\right\| \leq \mathcal{K}_{\gamma}, \quad\left\|\mathcal{I}_{\gamma}\left(z_{1}\left(t_{\gamma}^{-}\right)\right)-\mathcal{I}_{\gamma}\left(z_{2}\left(t_{\gamma}^{-}\right)\right)\right\| \leq \mathcal{D}_{\gamma}\left\|z_{1}\left(t_{\gamma}^{-}\right)-z_{2}\left(t_{\gamma}^{-}\right)\right\| .
$$

[X5]: The following inequalities hold

$$
\hat{\mathcal{O}}=\max _{1 \leq \gamma \leq \mathcal{H}}\left[\mathcal{M}_{1} \hat{\mathcal{K}}_{\mathcal{G}}, \mathcal{M}_{1}\left(1+\mathcal{D}_{\gamma}\right)\right]<1
$$

[X6]: There exists a constant $\hat{\mathcal{R}}_{\Delta}>0$ such that

$$
\left\|\Delta\left(t, z_{1}\right)-\Delta\left(t, z_{2}\right)\right\| \leq \hat{\mathcal{R}}_{\Delta}\left\|z_{1}-z_{2}\right\|, \forall z_{1}, z_{2} \in E .
$$

Theorem 1. Suppose the hypotheses [X1]-[X5] are fulfilled. If

$$
\mathcal{M}_{1} \hat{\mathcal{K}}_{\mathcal{G}}+\mathcal{M}_{2} \mathcal{K}_{\Delta} \frac{\Gamma\left(\sigma_{1}\right) \Gamma(\rho)}{\Gamma\left(\rho+\sigma_{1}\right)}(\varphi(b)-\varphi(0))^{\sigma_{1}}<1,
$$

then $\varphi$-fractional system (1) has at least one mild solution on $\mathcal{J}_{0}$.

Proof. For any $\pi>0$, we define

$$
\Omega_{\pi}=\left\{z \in \mathcal{P C}(E):\|z\|_{\mathcal{P C}} \leq \pi\right\}
$$

Clearly, $\Omega_{\pi}$ is closed convex and bounded subset of $\mathcal{P C}(E)$. Define an operator $\Pi: \Omega_{\pi} \rightarrow \mathcal{P C}(E)$ by

$$
(\Pi z)(t)= \begin{cases}\mathcal{S}_{\varphi}^{\sigma_{1}, \sigma_{2}}(t, 0)\left[z_{0}-\mathcal{G}(z)\right] & \\ +\int_{0}^{t}(\varphi(t)-\varphi(s))^{\sigma_{1}-1} \mathcal{T}_{\varphi}^{\sigma_{1}}(t, s) \Delta(s, z(s)) \varphi^{\prime}(s) d s, & t \in\left[0, t_{1}\right], \gamma=0, \\ \mathcal{S}_{\varphi}^{\sigma_{1}, \sigma_{2}}\left(t, t_{\gamma}\right)\left[z\left(t_{\gamma}^{-}\right)+\mathcal{I}_{\gamma}\left(z\left(t_{\gamma}^{-}\right)\right)\right] & \\ +\int_{t_{\gamma}}^{t}(\varphi(t)-\varphi(s))^{\sigma_{1}-1} \mathcal{T}_{\varphi}^{\sigma_{1}}(t, s) \Delta(s, z(s)) \varphi^{\prime}(s) d s, & t \in\left(t_{\gamma}, t_{\gamma+1}\right], \gamma \geq 1 .\end{cases}
$$

Now, we split $\Pi$ as $\Pi_{1}+\Pi_{2}$, where

$$
\begin{aligned}
& \left(\Pi_{1} z\right)(t)= \begin{cases}\mathcal{S}_{\varphi}^{\sigma_{1}, \sigma_{2}}(t, 0)\left[z_{0}-\mathcal{G}(z)\right], & t \in\left[0, t_{1}\right], \gamma=0, \\
\mathcal{S}_{\varphi}^{\sigma_{1}, \sigma_{2}}\left(t, t_{\gamma}\right)\left[z\left(t_{\gamma}^{-}\right)+\mathcal{I}_{\gamma}\left(z\left(t_{\gamma}^{-}\right)\right)\right], & t \in\left(t_{\gamma}, t_{\gamma+1}\right], \gamma \geq 1,\end{cases} \\
& \quad \text { and } \\
& \left(\Pi_{2} z\right)(t)= \begin{cases}\int_{0}^{t}(\varphi(t)-\varphi(s))^{\sigma_{1}-1} \mathcal{T}_{\varphi}^{\sigma_{1}}(t, s) \Delta(s, z(s)) \varphi^{\prime}(s) d s, & t \in\left[0, t_{1}\right], \gamma=0, \\
\int_{t_{\gamma}}^{t}(\varphi(t)-\varphi(s))^{\sigma_{1}-1} \mathcal{T}_{\varphi}^{\sigma_{1}}(t, s) \Delta(s, z(s)) \varphi^{\prime}(s) d s, & t \in\left(t_{\gamma}, t_{\gamma+1}\right], \gamma \geq 1 .\end{cases}
\end{aligned}
$$


Step 1. There exists $\pi>0$ such that $\Pi\left(\Omega_{\pi}\right) \subset \Omega_{\pi}$. If we assume that the assertion is not true, then for $\pi>0$, we take $t \in \mathcal{J}_{0}$ and $z^{\pi} \in \Omega_{\pi}$ such that $\left\|\Pi\left(z^{\pi}\right)\right\|_{\mathcal{P C}}>\pi$. For $t \in\left[0, t_{1}\right]$, we obtain

$$
\begin{aligned}
\pi<\left\|\Pi\left(z^{\pi}\right)\right\|_{\mathcal{P C}} \leq\left\|(\varphi(t)-\varphi(0))^{1-\rho} \mathcal{S}_{\varphi}^{\sigma_{1}, \sigma_{2}}(t, 0)\left[z_{0}-\mathcal{G}\left(z^{\pi}\right)\right]\right\| \\
+\left\|(\varphi(t)-\varphi(0))^{1-\rho} \int_{0}^{t}(\varphi(t)-\varphi(s))^{\sigma_{1}-1} \mathcal{T}_{\varphi}^{\sigma_{1}}(t, s) \Delta\left(s, z^{\pi}(s)\right) \varphi^{\prime}(s) d s\right\| \\
\leq \mathcal{M}_{1}\left[\left\|z_{0}\right\|_{\mathcal{P C}}+\hat{\mathcal{K}}_{\mathcal{G}} \pi+\|\mathcal{G}(0)\|_{\mathcal{P C}}\right] \\
+\mathcal{M}_{2} \mathcal{K}_{\Delta}\left(\varphi\left(t_{1}\right)-\varphi(0)\right)^{1-\rho} \int_{0}^{t}(\varphi(t)-\varphi(s))^{\sigma_{1}-1}\left\|z^{\pi}(s)\right\| \varphi^{\prime}(s) d s \\
\leq \mathcal{M}_{1}\left[\left\|z_{0}\right\|_{\mathcal{P C}}+\hat{\mathcal{K}}_{\mathcal{G}} \pi+\|\mathcal{G}(0)\|_{\mathcal{P C}}\right] \\
+\pi \mathcal{M}_{2} \mathcal{K}_{\Delta}\left(\varphi\left(t_{1}\right)-\varphi(0)\right)^{1-\rho} \int_{0}^{t}(\varphi(t)-\varphi(s))^{\sigma_{1}-1}(\varphi(s)-\varphi(0))^{\rho-1} \varphi^{\prime}(s) d s \\
\leq \mathcal{M}_{1}\left[\left\|z_{0}\right\|_{\mathcal{P C}}+\hat{\mathcal{K}}_{\mathcal{G}} \pi+\|\mathcal{G}(0)\|_{\mathcal{P C}}\right] \\
+\pi \mathcal{M}_{2} \mathcal{K}_{\Delta}\left(\varphi\left(t_{1}\right)-\varphi(0)\right)^{1-\rho} \Gamma\left(\sigma_{1}\right) I_{0^{+}}^{\sigma_{1}: \varphi}(\varphi(s)-\varphi(0))^{\rho-1} \\
\leq \mathcal{M}_{1}\left[\left\|z_{0}\right\|_{\mathcal{P C}}+\hat{\mathcal{K}}_{\mathcal{G}} \pi+\|\mathcal{G}(0)\|_{\mathcal{P C}}\right] \\
+\pi \mathcal{M}_{2} \mathcal{K}_{\Delta}\left(\varphi\left(t_{1}\right)-\varphi(0)\right)^{1-\rho} \frac{\Gamma\left(\sigma_{1}\right) \Gamma(\rho)}{\Gamma\left(\rho+\sigma_{1}\right)}\left(\varphi\left(t_{1}\right)-\varphi(0)\right)^{\rho+\sigma_{1}-1} \\
\leq \mathcal{M}_{1}\left[\left\|z_{0}\right\|_{\mathcal{P C}}+\hat{\mathcal{K}}_{\mathcal{G}} \pi+\|\mathcal{G}(0)\|_{\mathcal{P C}}\right] \\
+\pi \mathcal{M}_{2} \mathcal{K}_{\Delta} \frac{\Gamma\left(\sigma_{1}\right) \Gamma(\rho)}{\Gamma\left(\rho+\sigma_{1}\right)}\left(\varphi\left(t_{1}\right)-\varphi(0)\right)^{\sigma_{1}} .
\end{aligned}
$$

For every $t \in\left(t_{\gamma}, t_{\gamma+1}\right], \gamma=1,2 \ldots, \mathcal{H}$, we obtain

$$
\begin{aligned}
\pi<\left\|\Pi\left(z^{\pi}\right)\right\|_{\mathcal{P C}} & \leq\left\|\left(\varphi(t)-\varphi\left(t_{\gamma}\right)\right)^{1-\rho} \mathcal{S}_{\varphi}^{\sigma_{1}, \sigma_{2}}\left(t, t_{\gamma}\right)\left[z^{\pi}\left(t_{\gamma}^{-}\right)+\mathcal{I}_{\gamma}\left(z^{\pi}\left(t_{\gamma}^{-}\right)\right)\right]\right\| \\
& +\left\|\left(\varphi(t)-\varphi\left(t_{\gamma}\right)\right)^{1-\rho} \int_{t_{\gamma}}^{t}(\varphi(t)-\varphi(s))^{\sigma_{1}-1} \mathcal{T}_{\varphi}^{\sigma_{1}}(t, s) \Delta\left(s, z^{\pi}(s)\right) \varphi^{\prime}(s) d s\right\| \\
& \leq \mathcal{M}_{1}\left[\left\|z^{\pi}\left(t_{\gamma}^{-}\right)\right\|_{\mathcal{P C}}+\left(\varphi\left(t_{\gamma+1}\right)-\varphi\left(t_{\gamma}\right)\right)^{1-\rho} \mathcal{K}_{\gamma}\right] \\
& +\mathcal{M}_{2} \mathcal{K}_{\Delta}\left(\varphi\left(t_{\gamma+1}\right)-\varphi\left(t_{\gamma}\right)\right)^{1-\rho} \int_{t_{\gamma}}^{t}(\varphi(t)-\varphi(s))^{\sigma_{1}-1}\left\|z^{\pi}(s)\right\| \varphi^{\prime}(s) d s \\
& \leq \mathcal{M}_{1}\left[\left\|z^{\pi}\left(t_{\gamma}^{-}\right)\right\|_{\mathcal{P C}}+\left(\varphi\left(t_{\gamma+1}\right)-\varphi\left(t_{\gamma}\right)\right)^{1-\rho} \mathcal{K}_{\gamma}\right] \\
& +\pi \mathcal{M}_{2} \mathcal{K}_{\Delta}\left(\varphi\left(t_{\gamma+1}\right)-\varphi\left(t_{\gamma}\right)\right)^{1-\rho} \int_{t_{\gamma}}^{t}(\varphi(t)-\varphi(s))^{\sigma_{1}-1}\left(\varphi(s)-\varphi\left(t_{\gamma}\right)\right)^{\rho-1} \varphi^{\prime}(s) d s \\
& \leq \mathcal{M}_{1}\left[\left\|z^{\pi}\left(t_{\gamma}^{-}\right)\right\|_{\mathcal{P C}}+\left(\varphi\left(t_{\gamma+1}\right)-\varphi\left(t_{\gamma}\right)\right)^{1-\rho} \mathcal{K}_{\gamma}\right] \\
& +\pi \mathcal{M}_{2} \mathcal{K}_{\Delta}\left(\varphi\left(t_{\gamma+1}\right)-\varphi\left(t_{\gamma}\right)\right)^{1-\rho} \Gamma\left(\sigma_{1}\right) I_{t_{\gamma}^{+}}^{\sigma_{1}: \varphi}\left(\varphi(s)-\varphi\left(t_{\gamma}\right)\right)^{\rho-1} \\
& \leq \mathcal{M}_{1}\left[\left\|z^{\pi}\left(t_{\gamma}^{-}\right)\right\|_{\mathcal{P C}}+\left(\varphi\left(t_{\gamma+1}\right)-\varphi\left(t_{\gamma}\right)\right)^{1-\rho} \mathcal{K}_{\gamma}\right] \\
& +\pi \mathcal{M}_{2} \mathcal{K}_{\Delta}\left(\varphi\left(t_{\gamma+1}\right)-\varphi\left(t_{\gamma}\right)\right)^{1-\rho} \frac{\Gamma\left(\sigma_{1}\right) \Gamma(\rho)}{\Gamma\left(\rho+\sigma_{1}\right)}\left(\varphi\left(t_{\gamma+1}\right)-\varphi\left(t_{\gamma}\right)\right)^{\rho+\sigma_{1}-1} \\
& \leq \mathcal{M}_{1}\left[\left\|z^{\pi}\left(t_{\gamma}^{-}\right)\right\|_{\mathcal{P C}}+\left(\varphi\left(t_{\gamma+1}\right)-\varphi\left(t_{\gamma}\right)\right)^{1-\rho} \mathcal{K}_{\gamma}\right] \\
& +\pi \mathcal{M}_{2} \mathcal{K}_{\Delta} \frac{\Gamma\left(\sigma_{1}\right) \Gamma(\rho)}{\Gamma\left(\rho+\sigma_{1}\right)}\left(\varphi\left(t_{\gamma+1}\right)-\varphi\left(t_{\gamma}\right)\right)^{\sigma_{1}} .
\end{aligned}
$$

For every $t \in \mathcal{J}_{0}$, we obtain

$$
\pi<\left\|\Pi\left(z^{\pi}\right)\right\|_{\mathcal{P C}} \leq \mathcal{W}^{*}+\mathcal{M}_{1} \hat{\mathcal{K}}_{\mathcal{G}} \pi+\pi \mathcal{M}_{2} \mathcal{K}_{\Delta} \frac{\Gamma\left(\sigma_{1}\right) \Gamma(\rho)}{\Gamma\left(\rho+\sigma_{1}\right)}(\varphi(b)-\varphi(0))^{\sigma_{1}},
$$


where

$$
\mathcal{W}^{*}=\max _{1 \leq \gamma \leq \mathcal{H}}\left\{\mathcal{M}_{1}\left[\left\|z_{0}\right\|_{\mathcal{P C}}+\|\mathcal{G}(0)\|_{\mathcal{P C}}\right]+\mathcal{M}_{1}\left[\left\|z^{\pi}\left(t_{\gamma}^{-}\right)\right\|_{\mathcal{P C}}+\left(\varphi\left(t_{\gamma+1}\right)-\varphi\left(t_{\gamma}\right)\right)^{1-\rho} \mathcal{K}_{\gamma}\right]\right\} .
$$

Here, $\mathcal{W}^{*}$ is independent of $\pi$, both sides of Equation (7) are dividing by $\pi$ and taking $\pi \rightarrow \infty$, we obtain

$$
1<\mathcal{M}_{1} \hat{\mathcal{K}}_{\mathcal{G}}+\mathcal{M}_{2} \mathcal{K}_{\Delta} \frac{\Gamma\left(\sigma_{1}\right) \Gamma(\rho)}{\Gamma\left(\rho+\sigma_{1}\right)}(\varphi(b)-\varphi(0))^{\sigma_{1}}
$$

which contradicts to Equation (6). Hence, for some $\pi>0, \Pi\left(\Omega_{\pi}\right) \subset \Omega_{\pi}$.

Step 2. We will prove that $\Pi_{1}$ is a contraction map.

For $z^{*}, z^{* *} \in \Omega_{\pi}$, if $t \in\left[0, t_{1}\right]$, then we obtain

$$
\begin{aligned}
\left\|\Pi_{1} z^{*}-\Pi_{1} z^{* *}\right\|_{\mathcal{P C}} & =\left\|(\varphi(t)-\varphi(0))^{1-\rho} \mathcal{S}_{\varphi}^{\sigma_{1}, \sigma_{2}}(t, 0)\left[\mathcal{G}\left(z^{*}\right)-\mathcal{G}\left(z^{* *}\right)\right]\right\| \\
& \leq \mathcal{M}_{1} \hat{\mathcal{K}}_{\mathcal{G}}\left\|z^{*}-z^{* *}\right\|_{\mathcal{P C}} .
\end{aligned}
$$

Similarly, if $t \in\left(t_{\gamma}, t_{\gamma+1}\right], \gamma=1,2, \ldots, \mathcal{H}$, then we get

$$
\begin{aligned}
\left\|\Pi_{1} z^{*}-\Pi_{1} z^{* *}\right\|_{\mathcal{P C}} & =\left\|\left(\varphi(t)-\varphi\left(t_{\gamma}\right)\right)^{1-\rho} \mathcal{S}_{\varphi}^{\sigma_{1}, \sigma_{2}}\left(t, t_{\gamma}\right)\left[z^{*}\left(t_{\gamma}^{-}\right)-z^{* *}\left(t_{\gamma}^{-}\right)\right]\right\| \\
& +\left\|\left(\varphi(t)-\varphi\left(t_{\gamma}\right)\right)^{1-\rho} \mathcal{S}_{\varphi}^{\sigma_{1}, \sigma_{2}}\left(t, t_{\gamma}\right)\left[\mathcal{I}_{\gamma}\left(z^{*}\left(t_{\gamma}^{-}\right)\right)-\mathcal{I}_{\gamma}\left(z^{* *}\left(t_{\gamma}^{-}\right)\right)\right]\right\| \\
& \leq \mathcal{M}_{1}\left(1+\mathcal{D}_{\gamma}\right)\left\|z^{*}-z^{* *}\right\|_{\mathcal{P C}} .
\end{aligned}
$$

From Equations (8) and (9), we obtain

$$
\left\|\Pi_{1} z^{*}-\Pi_{1} z^{* *}\right\|_{\mathcal{P C}} \leq \hat{\mathcal{O}}\left\|z^{*}-z^{* *}\right\|_{\mathcal{P C}},
$$

where $\hat{\mathcal{O}}=\max _{1 \leq \gamma \leq \mathcal{H}}\left[\mathcal{M}_{1} \hat{\mathcal{K}}_{\mathcal{G}}, \mathcal{M}_{1}\left(1+\mathcal{D}_{\gamma}\right)\right]$. By [X5], we see that $\hat{\mathcal{O}}<1$. Hence, $\Pi_{1}$ is a contraction mapping.

Step 3. We will prove that $\Pi_{2}: \Omega_{\pi} \rightarrow \Omega_{\pi}$ is continuous.

Let $\left\{z_{k}\right\} \subset \Omega_{\pi}$ with $z_{k} \rightarrow z$ as $k \rightarrow \infty$. By [X2], we obtain

$$
\Delta\left(t, z_{k}\right) \rightarrow \Delta(t, z) \text { as } k \rightarrow \infty,
$$

and

$$
\left\|\Delta\left(t, z_{k}(t)\right)-\Delta(t, z(t))\right\| \leq 2 \hat{\mathcal{K}}_{\Delta}(t) \pi .
$$

For every $t \in\left(t_{\gamma}, t_{\gamma+1}\right], \gamma=0,1, \ldots, \mathcal{H}$, we obtain

$$
\begin{aligned}
\left\|\Pi_{2}\left(z_{k}\right)-\Pi_{2}(z)\right\|_{\mathcal{P C}} & \leq \|\left(\varphi(t)-\varphi\left(t_{\gamma}\right)\right)^{1-\rho} \int_{t_{\gamma}}^{t}(\varphi(t)-\varphi(s))^{\sigma_{1}-1} \mathcal{T}_{\varphi}^{\sigma_{1}}(t, s) \\
& \times\left[\Delta\left(s, z_{k}(s)\right)-\Delta(s, z(s))\right] \varphi^{\prime}(s) d s \| \\
& \leq \mathcal{M}_{2}\left(\varphi\left(t_{\gamma+1}\right)-\varphi\left(t_{\gamma}\right)\right)^{1-\rho} \\
& \times \int_{t_{\gamma}}^{t}(\varphi(t)-\varphi(s))^{\sigma_{1}-1}\left\|\Delta\left(s, z_{k}(s)\right)-\Delta(s, z(s))\right\| \varphi^{\prime}(s) d s .
\end{aligned}
$$

By the Lebesgue dominated convergence theorem, we obtain

$$
\left\|\Pi_{2}\left(z_{k}\right)-\Pi_{2}(z)\right\|_{\mathcal{P C}} \rightarrow 0 \text { as } k \rightarrow \infty .
$$

Hence, $\Pi_{2}$ is continuous.

Step 4 . We prove that $\left\{\Pi_{2} z: z \in \Omega_{\pi}\right\}$ is equicontinuous. 
Let $\kappa_{1}, \kappa_{2} \in\left(t_{\gamma}, t_{\gamma+1}\right]$, with $t_{\gamma}<\kappa_{1}<\kappa_{2} \leq t_{\gamma+1}$, then we obtain for every $t \in$ $\left(t_{\gamma}, t_{\gamma+1}\right], \gamma=0,1, \ldots, \mathcal{H}$

$$
\begin{aligned}
\|\left(\varphi\left(\kappa_{2}\right)-\right. & \left.\varphi\left(t_{\gamma}\right)\right)^{1-\rho}\left(\Pi_{2} z\right)\left(\kappa_{2}\right)-\left(\varphi\left(\kappa_{1}\right)-\varphi\left(t_{\gamma}\right)\right)^{1-\rho}\left(\Pi_{2} z\right)\left(\kappa_{1}\right) \| \\
& \leq \int_{t_{\gamma}}^{\kappa_{1}} \|\left(\varphi\left(\kappa_{2}\right)-\varphi\left(t_{\gamma}\right)\right)^{1-\rho}\left(\varphi\left(\kappa_{2}\right)-\varphi(s)\right)^{\sigma_{1}-1} \mathcal{T}_{\varphi}^{\sigma_{1}}\left(\kappa_{2}, s\right) \\
& -\left(\varphi\left(\kappa_{1}\right)-\varphi\left(t_{\gamma}\right)\right)^{1-\rho}\left(\varphi\left(\kappa_{1}\right)-\varphi(s)\right)^{\sigma_{1}-1} \mathcal{T}_{\varphi}^{\sigma_{1}}\left(\kappa_{1}, s\right)\|\| \Delta(s, z(s)) \| \varphi^{\prime}(s) d s \\
& +\int_{\kappa_{1}}^{\kappa_{2}}\left\|\left(\varphi\left(\kappa_{2}\right)-\varphi\left(t_{\gamma}\right)\right)^{1-\rho}\left(\varphi\left(\kappa_{2}\right)-\varphi(s)\right)^{\sigma_{1}-1} \mathcal{T}_{\varphi}^{\sigma_{1}}\left(\kappa_{2}, s\right)\right\|\|\Delta(s, z(s))\| \varphi^{\prime}(s) d s .
\end{aligned}
$$

As $\kappa_{2} \rightarrow \kappa_{1}$, the right-hand side of Equation (10) tends to zero. Thus, the equicontinuity of $\left\{\Pi_{2} z: z \in \Omega_{\pi}\right\}$ is obtained.

Step 5 . We prove that $\delta(t)=\left\{\left(\Pi_{2} z\right)(t): z \in \Omega_{\pi}\right\}$ is relatively compact in $E$.

Obviously, $\delta(0)=\{0\}$ is relatively compact. Let $t \in\left(t_{\gamma}, t_{\gamma+1}\right]$ be fixed, $0<\epsilon<t$, and $\epsilon$ is real number. For $z \in \Omega_{\pi}$, we define

$$
\left(\Pi_{2}^{\epsilon} z\right)(t)= \begin{cases}\int_{0}^{t-\epsilon}(\varphi(t)-\varphi(s))^{\sigma_{1}-1} \mathcal{T}_{\varphi}^{\sigma_{1}}(t, s) \Delta(s, z(s)) \varphi^{\prime}(s) d s, & t \in\left[0, t_{1}\right], \gamma=0, \\ \int_{t_{\gamma}}^{t-\epsilon}(\varphi(t)-\varphi(s))^{\sigma_{1}-1} \mathcal{T}_{\varphi}^{\sigma_{1}}(t, s) \Delta(s, z(s)) \varphi^{\prime}(s) d s, & t \in\left(t_{\gamma}, t_{\gamma+1}\right], \gamma \geq 1 .\end{cases}
$$

By [X1], we obtain $\delta^{\epsilon}(t)=\left\{\left(\Pi^{\epsilon} z\right)(t): z \in \Omega_{\pi}\right\}$ is relatively compact in $E$. for every $z \in \Omega_{\pi}$, we get

$$
\begin{aligned}
\left\|\left(\varphi(t)-\varphi\left(t_{\gamma}\right)\right)^{1-\rho}\left[\left(\Pi_{2} z\right)(t)-\left(\Pi_{2}^{\epsilon} z\right)(t)\right]\right\| & \leq \pi \mathcal{M}_{2} \mathcal{K}_{\Delta}\left(\varphi\left(t_{\gamma+1}\right)-\varphi\left(t_{\gamma}\right)\right)^{1-\rho} \\
& \times \int_{t-\epsilon}^{t}(\varphi(t)-\varphi(s))^{\sigma_{1}-1}\left(\varphi(s)-\varphi\left(t_{\gamma}\right)\right)^{\rho-1} \varphi^{\prime}(s) d s \\
& \rightarrow 0 \text { as } \epsilon \rightarrow 0 .
\end{aligned}
$$

Then $\delta(t)$ is relatively compact in E. By steps $3-5$ and Arzela-Ascoli theorem, $\Pi_{2}$ is completely continuous. Hence, by the fixed point theorem of Krasnoselskii's [33], there exists at least one mild solution on $\mathcal{J}_{0}$.

Theorem 2. Suppose the hypotheses [X1]-[X6] are fulfilled. Then $\varphi$-fractional system (1) has a unique mild solution on $\mathcal{J}_{0}$.

Proof. Let $z_{1}$ and $z_{2}$ be the mild solutions of the $\varphi$-fractional system (1) in $\Omega_{\pi}$. Then, for each $k \in\{1,2\}$, the mild solutions $z_{k}$ satisfies

$$
\left(\Pi z_{k}\right)(t)= \begin{cases}\mathcal{S}_{\varphi}^{\sigma_{1}, \sigma_{2}}(t, 0)\left[z_{0}-\mathcal{G}\left(z_{k}\right)\right] & \\ +\int_{0}^{t}(\varphi(t)-\varphi(s))^{\sigma_{1}-1} \mathcal{T}_{\varphi}^{\sigma_{1}}(t, s) \Delta\left(s, z_{k}(s)\right) \varphi^{\prime}(s) d s, & t \in\left[0, t_{1}\right], \gamma=0, \\ \mathcal{S}_{\varphi}^{\sigma_{1}, \sigma_{2}}\left(t, t_{\gamma}\right)\left[z_{k}\left(t_{\gamma}^{-}\right)+\mathcal{I}_{\gamma}\left(z_{k}\left(t_{\gamma}^{-}\right)\right)\right] & \\ +\int_{t_{\gamma}}^{t}(\varphi(t)-\varphi(s))^{\sigma_{1}-1} \mathcal{T}_{\varphi}^{\sigma_{1}}(t, s) \Delta\left(s, z_{k}(s)\right) \varphi^{\prime}(s) d s, & t \in\left(t_{\gamma}, t_{\gamma+1}\right], \gamma \geq 1 .\end{cases}
$$

For every $t \in\left[0, t_{1}\right], \gamma=0$, we obtain 


$$
\begin{aligned}
\left\|(\varphi(t)-\varphi(0))^{1-\rho}\left[z_{1}(t)-z_{2}(t)\right]\right\| & =\left\|(\varphi(t)-\varphi(0))^{1-\rho}\left[\left(\Pi z_{1}\right)(t)-\left(\Pi z_{2}\right)(t)\right]\right\| \\
& \leq \mathcal{M}_{1} \hat{\mathcal{K}}_{\mathcal{G}}\left\|(\varphi(t)-\varphi(0))^{1-\rho}\left[z_{1}(t)-z_{2}(t)\right]\right\| \\
& +\mathcal{M}_{2} \hat{\mathcal{R}}_{\Delta}\left(\varphi\left(t_{1}\right)-\varphi(0)\right)^{1-\rho} \int_{0}^{t}(\varphi(t)-\varphi(s))^{\sigma_{1}-1} \\
& \times(\varphi(s)-\varphi(0))^{\rho-1}\left\|(\varphi(s)-\varphi(0))^{1-\rho}\left[z_{1}(s)-z_{2}(s)\right]\right\| \varphi^{\prime}(s) d s \\
& \leq \mathcal{M}_{1} \hat{\mathcal{K}}_{\mathcal{G}}\left\|(\varphi(t)-\varphi(0))^{1-\rho}\left[z_{1}(t)-z_{2}(t)\right]\right\| \\
& +\mathcal{M}_{2} \hat{\mathcal{R}}_{\Delta} K_{0}^{*}\left(\varphi\left(t_{1}\right)-\varphi(0)\right)^{1-\rho} \int_{0}^{t}(\varphi(t)-\varphi(s))^{\sigma_{1}-1} \\
& \times\left\|(\varphi(s)-\varphi(0))^{1-\rho}\left[z_{1}(s)-z_{2}(s)\right]\right\| \varphi^{\prime}(s) d s,
\end{aligned}
$$

where $K_{0}^{*}=\sup _{0 \leq s \leq t_{1}}(\varphi(s)-\varphi(0))^{\rho-1}$.

Then we obtain

$$
\begin{aligned}
\left\|(\varphi(t)-\varphi(0))^{1-\rho}\left[z_{1}(t)-z_{2}(t)\right]\right\| & \leq \frac{\mathcal{M}_{2} \hat{\mathcal{R}}_{\Delta} K_{0}^{*}\left(\varphi\left(t_{1}\right)-\varphi(0)\right)^{1-\rho}}{\left(1-\mathcal{M}_{1} \hat{\mathcal{K}}_{\mathcal{G}}\right)} \int_{0}^{t}(\varphi(t)-\varphi(s))^{\sigma_{1}-1} \\
& \times\left\|(\varphi(s)-\varphi(0))^{1-\rho}\left[z_{1}(s)-z_{2}(s)\right]\right\| \varphi^{\prime}(s) d s,
\end{aligned}
$$

where $\mathcal{M}_{1} \hat{\mathcal{K}}_{\mathcal{G}}<1$.

For every $t \in\left(t_{\gamma}, t_{\gamma+1}\right], \gamma=1,2, \ldots, \mathcal{H}$, we get

$$
\begin{aligned}
\left\|\left(\varphi(t)-\varphi\left(t_{\gamma}\right)\right)^{1-\rho}\left[z_{1}(t)-z_{2}(t)\right]\right\| & =\left\|\left(\varphi(t)-\varphi\left(t_{\gamma}\right)\right)^{1-\rho}\left[\left(\Pi z_{1}\right)(t)-\left(\Pi z_{2}\right)(t)\right]\right\| \\
& \leq \mathcal{M}_{1}\left(1+\mathcal{D}_{\gamma}\right)\left\|\left(\varphi(t)-\varphi\left(t_{\gamma}\right)\right)^{1-\rho}\left[z_{1}\left(t_{\gamma}^{-}\right)-z_{2}\left(t_{\gamma}^{-}\right)\right]\right\| \\
& +\mathcal{M}_{2} \hat{\mathcal{R}}_{\Delta}\left(\varphi\left(t_{\gamma+1}\right)-\varphi\left(t_{\gamma}\right)\right)^{1-\rho} \int_{t_{\gamma}}^{t}(\varphi(t)-\varphi(s))^{\sigma_{1}-1} \\
& \times\left(\varphi(s)-\varphi\left(t_{\gamma}\right)\right)^{\rho-1}\left\|\left(\varphi(s)-\varphi\left(t_{\gamma}\right)\right)^{1-\rho}\left[z_{1}(s)-z_{2}(s)\right]\right\| \varphi^{\prime}(s) d s \\
& \leq \mathcal{M}_{1}\left(1+\mathcal{D}_{\gamma}\right)\left\|\left(\varphi(t)-\varphi\left(t_{\gamma}\right)\right)^{1-\rho}\left[z_{1}\left(t_{\gamma}^{-}\right)-z_{2}\left(t_{\gamma}^{-}\right)\right]\right\| \\
& +\mathcal{M}_{2} \hat{\mathcal{R}}_{\Delta} K_{\gamma}^{*}\left(\varphi\left(t_{\gamma+1}\right)-\varphi\left(t_{\gamma}\right)\right)^{1-\rho} \int_{t_{\gamma}}^{t}(\varphi(t)-\varphi(s))^{\sigma_{1}-1} \\
& \times\left\|\left(\varphi(s)-\varphi\left(t_{\gamma}\right)\right)^{1-\rho}\left[z_{1}(s)-z_{2}(s)\right]\right\| \varphi^{\prime}(s) d s,
\end{aligned}
$$

where $K_{\gamma}^{*}=\sup _{t_{\gamma} \leq s \leq t_{\gamma+1}}(\varphi(s)-\varphi(0))^{\rho-1}, \gamma=1,2, \ldots, \mathcal{H}$.

Then we obtain

$$
\begin{aligned}
\left\|\left(\varphi(t)-\varphi\left(t_{\gamma}\right)\right)^{1-\rho}\left[z_{1}(t)-z_{2}(t)\right]\right\| & \leq \frac{\mathcal{M}_{2} \hat{\mathcal{R}}_{\Delta} K_{\gamma}^{*}\left(\varphi\left(t_{\gamma+1}\right)-\varphi\left(t_{\gamma}\right)\right)^{1-\rho}}{\left(1-\mathcal{M}_{1}\left(1+\mathcal{D}_{\gamma}\right)\right)} \int_{t_{\gamma}}^{t}(\varphi(t)-\varphi(s))^{\sigma_{1}-1} \\
& \times\left\|\left(\varphi(s)-\varphi\left(t_{\gamma}\right)\right)^{1-\rho}\left[z_{1}(s)-z_{2}(s)\right]\right\| \varphi^{\prime}(s) d s,
\end{aligned}
$$

where $\mathcal{M}_{1}\left(1+\mathcal{D}_{\gamma}\right)<1$.

By using the Gronwall's inequality (Theorem 2.11, [17]), we get

$$
\left\|z_{1}-z_{2}\right\|_{\mathcal{P C}}=0,
$$

which implies that $z_{1} \equiv z_{2}$. Therefore, $\varphi$-fractional system (1) has a unique mild solution on $\mathcal{J}_{0}$. 


\section{Existence of Optimal Controls}

Let $v$ takes the value in the separable reflexive Banach space $\mathcal{T}$ and $\mathcal{V}_{f}(\mathcal{T})$ is a class of subsets of $\mathcal{T}$, which is nonempty convex and closed. The multifunction $g: \mathcal{J} \rightarrow \mathcal{V}_{f}(\mathcal{T})$ is measurable and $g(\cdot) \subset \triangle$, the admissible control set

$$
\mathcal{U}_{a d}=\left\{v \in L^{2}(\triangle): v(t) \in g(t) \text { a.e. }\right\},
$$

where $\triangle$ is a bounded set of $\mathcal{T}$. Then $\mathcal{U}_{a d} \neq \phi$.

Consider following $\varphi$-Hilfer fractional impulsive differential control system:

$$
\left\{\begin{array}{l}
{ }^{H} \mathrm{D}_{t_{\gamma}}^{\sigma_{1}, \sigma_{2}: \varphi} z(t)=\mathcal{A} z(t)+\mathcal{D} v(t)+\Delta(t, z(t)), t \in(0, b]-\left\{t_{1}, t_{2}, \ldots, t_{\mathcal{H}}\right\}, \\
I_{t_{\gamma}^{+}}^{\left(1-\sigma_{1}\right)\left(1-\sigma_{2}\right) ; \varphi} z\left(t_{\gamma}^{+}\right)=z\left(t_{\gamma}^{-}\right)+\mathcal{I}_{\gamma}\left(z\left(t_{\gamma}^{-}\right)\right), \gamma=1,2, \ldots, \mathcal{H}, \\
I_{0^{+}}^{\left(1-\sigma_{1}\right)\left(1-\sigma_{2}\right) ; \varphi}[z(t)]_{t=0}+\mathcal{G}(z)=z_{0} .
\end{array}\right.
$$

Let us assume the following hypotheses

[X7]: $\mathcal{D} \in L^{\infty}\left(\mathcal{J}_{0}, L(\mathcal{T}, E)\right)$, that implies that $\mathcal{D} v \in L^{2}\left(\mathcal{J}_{0}, E\right)$ for $v \in \mathcal{U}_{a d}$.

$[\mathrm{X} 8]: \mathcal{K}_{*}=\sup _{t \in \mathcal{J}_{0}} \varphi^{\prime}(t)<\infty$.

Theorem 3. Suppose the hypotheses of Theorem 2 and [X7]-[X8] are fulfilled. Then for each $v \in \mathcal{U}_{a d}, \varphi$-fractional system (11) has a mild solution which is given by

$$
z^{v}(t)= \begin{cases}\mathcal{S}_{\varphi}^{\sigma_{1}, \sigma_{2}}(t, 0)\left[z_{0}-\mathcal{G}(z)\right] & t \in\left[0, t_{1}\right], \gamma=0, \\ +\int_{0}^{t}(\varphi(t)-\varphi(s))^{\sigma_{1}-1} \mathcal{T}_{\varphi}^{\sigma_{1}}(t, s)[\mathcal{D} v(s)+\Delta(s, z(s))] \varphi^{\prime}(s) d s, & \\ \mathcal{S}_{\varphi}^{\sigma_{1}, \sigma_{2}}\left(t, t_{\gamma}\right)\left[z\left(t_{\gamma}^{-}\right)+\mathcal{I}_{\gamma}\left(z\left(t_{\gamma}^{-}\right)\right)\right] & t \in\left(t_{\gamma}, t_{\gamma+1}\right], \gamma \geq 1 . \\ +\int_{t_{\gamma}}^{t}(\varphi(t)-\varphi(s))^{\sigma_{1}-1} \mathcal{T}_{\varphi}^{\sigma_{1}}(t, s)[\mathcal{D} v(s)+\Delta(s, z(s))] \varphi^{\prime}(s) d s, & \end{cases}
$$

Proof. Let us consider

$$
\mathcal{H}(t)=\int_{t_{\gamma}}^{t}(\varphi(t)-\varphi(s))^{\sigma_{1}-1} \mathcal{T}_{\varphi}^{\sigma_{1}}(t, s) \mathcal{D} v(s) \varphi^{\prime}(s) d s .
$$

By Hölder's inequality and [X7], we get

$$
\begin{aligned}
\left\|\left(\varphi(t)-\varphi\left(t_{\gamma}\right)\right)^{1-\rho} \mathcal{H}(t)\right\| & \leq \mathcal{M}_{2}\|\mathcal{D}\|_{\infty}\left(\varphi\left(t_{\gamma+1}\right)-\varphi\left(t_{\gamma}\right)\right)^{1-\rho} \int_{t_{\gamma}}^{t}(\varphi(t)-\varphi(s))^{\sigma_{1}-1}\|v(s)\|_{\mathcal{T}} \varphi^{\prime}(s) d s \\
& \leq \mathcal{M}_{2}\|\mathcal{D}\|_{\infty}\left(\varphi\left(t_{\gamma+1}\right)-\varphi\left(t_{\gamma}\right)\right)^{1-\rho} \\
& \times\left(\int_{t_{\gamma}}^{t}(\varphi(t)-\varphi(s))^{2\left(\sigma_{1}-1\right)} \varphi^{\prime}(s) d s\right)^{1 / 2}\left(\int_{t_{\gamma}}^{t}\|v(s)\|_{\mathcal{T}}^{2} \varphi^{\prime}(s) d s\right)^{1 / 2} \\
& \leq \frac{\mathcal{M}_{2}\|\mathcal{D}\|_{\infty}\left(\varphi\left(t_{\gamma+1}\right)-\varphi\left(t_{\gamma}\right)\right)^{\sigma_{1}-\rho+(1 / 2)}}{\left(2 \sigma_{1}-1\right)^{1 / 2}}\left(\int_{t_{\gamma}}^{t}\|v(s)\|_{\mathcal{T}}^{2} \varphi^{\prime}(s) d s\right)^{1 / 2} \\
& \leq \frac{\mathcal{M}_{2} \mathcal{K}_{*}^{1 / 2}\|\mathcal{D}\|_{\infty}\left(\varphi\left(t_{\gamma+1}\right)-\varphi\left(t_{\gamma}\right)\right)^{\sigma_{1}-\rho+(1 / 2)}}{\left(2 \sigma_{1}-1\right)^{1 / 2}}\|v\|_{L^{2}\left(\mathcal{J}_{0}, \mathcal{T}\right)} .
\end{aligned}
$$

It follows that $(\varphi(t)-\varphi(s))^{\sigma_{1}-1} \mathcal{T}_{\varphi}^{\sigma_{1}}(t, s) \mathcal{D} v(s) \varphi^{\prime}(s) d s$ are integrable on $\mathcal{J}_{0}$, here, $\|\mathcal{D}\|_{\infty}$ is the norm of $\mathcal{D}$ in Banach space $L^{\infty}\left(\mathcal{J}_{0}, L(\mathcal{T}, E)\right)$. Hence, $\mathcal{H}(\cdot) \in \Omega_{\pi}$. Using Theorem 2, we get the required results.

We consider the Lagrange problem

$$
(\mathcal{L P})\left\{\begin{array}{l}
\text { Find }\left(z^{*}, v^{*}\right) \in \mathcal{P C}(E) \times \mathcal{U}_{a d} \\
\text { such that } \mathcal{J}\left(z^{*}, v^{*}\right) \leq \mathcal{J}\left(z^{v}, v\right),\left(z^{v}, v\right) \in \mathcal{P C}(E) \times \mathcal{U}_{a d},
\end{array}\right.
$$


where the cost functional is

$$
\mathcal{J}\left(z^{v}, v\right)=\sum_{\gamma=0}^{\mathcal{H}} \int_{t_{\gamma}}^{t_{\gamma+1}} \mathcal{L}\left(t, z^{v}(t), v(t)\right) d t,
$$

where $z^{v}$ be the mild solution of (11) with respect to control $v \in \mathcal{U}_{a d}$.

Next, we assume

[X9]: 1. The functional $\mathcal{L}: \mathcal{J}_{0} \times E \times \mathcal{T} \rightarrow \mathbb{R} \cup\{\infty\}$ is Borel measurable.

2. For almost all $t \in \mathcal{J}_{0}, \mathcal{L}(t, \cdot, \cdot)$ is sequentially lower semicontinuous on $E \times \mathcal{T}$.

3. For each $z^{v} \in E$ and almost all $t \in \mathcal{J}_{0}, \mathcal{L}\left(t, z^{v}, \cdot\right)$ is convex on $\mathcal{T}$.

4. There exist constants $d_{1} \geq 0, d_{2}>0, \phi$ is non-negative function in $L^{1}\left(\mathcal{J}_{0}, \mathbb{R}\right)$ such that

$$
\mathcal{L}\left(t, z^{v}, v\right) \geq \phi(t)+d_{1}\left\|z^{v}\right\|+d_{2}\|v\|_{\mathcal{T}}^{2} .
$$

$[\mathrm{X} 10]: \mathcal{D}$ is a strongly continuous operator.

Theorem 4. If the assumptions [X1]-[X10] are fulfilled, then the problem $(\mathcal{L P})$ admits at least one optimal pair.

Proof. Assume that $\inf \left\{\mathcal{J}\left(z^{v}, v\right): v \in \mathcal{U}_{a d}\right\}=\epsilon<+\infty$. By using [X9], we obtain $\epsilon>-\infty$. By definition of infimum there exists a minimizing sequence feasible pair $\left(z^{k}, v^{k}\right) \subset \mathcal{P}_{a d}$, where $\mathcal{P}_{a d}=\left\{\left(z^{v}, v\right): z^{v}\right.$ is a solution of (11) with respect to $\left.v \in \mathcal{U}_{a d}\right\}$ such that $\mathcal{J}\left(z^{k}, v^{k}\right) \rightarrow$ $\epsilon$ as $k \rightarrow+\infty$. Since $v^{k} \subseteq \mathcal{U}_{a d}, v^{k}$ is bounded in $L^{2}\left(\mathcal{J}_{0}, \mathcal{T}\right)$, there exists a subsequence which is still represented by $v^{k}$ and $v^{*} \in L^{2}\left(\mathcal{J}_{0}, \mathcal{T}\right)$ such that

$$
v^{k} \stackrel{\mathrm{w}}{\longrightarrow} v^{*}
$$

in $L^{2}\left(\mathcal{J}_{0}, \mathcal{T}\right)$. Since $\mathcal{U}_{a d}$ is convex and closed, by using Marzur Lemma, we get $v^{*} \in \mathcal{U}_{a d}$. Let $z^{k}$ and $z^{*}$ be the mild solution of system (11) with respect to $v^{k}$ and $v^{*}$, respectively

$$
\begin{aligned}
& z^{k}(t)= \begin{cases}\mathcal{S}_{\varphi}^{\sigma_{1}, \sigma_{2}}(t, 0)\left[z_{0}-\mathcal{G}\left(z^{k}\right)\right] \\
+\int_{0}^{t}(\varphi(t)-\varphi(s))^{\sigma_{1}-1} \mathcal{T}_{\varphi}^{\sigma_{1}}(t, s)\left[\mathcal{D} v^{k}(s)+\Delta\left(s, z^{k}(s)\right)\right] \varphi^{\prime}(s) d s, & t \in\left[0, t_{1}\right], \gamma=0, \\
\mathcal{S}_{\varphi}^{\sigma_{1}, \sigma_{2}}\left(t, t_{\gamma}\right)\left[z^{k}\left(t_{\gamma}^{-}\right)+\mathcal{I}_{\gamma}\left(z^{k}\left(t_{\gamma}^{-}\right)\right]\right. & t \in\left(t_{\gamma}, t_{\gamma+1}\right], \gamma \geq 1, \\
+\int_{t_{\gamma}}^{t}(\varphi(t)-\varphi(s))^{\sigma_{1}-1} \mathcal{T}_{\varphi}^{\sigma_{1}}(t, s)\left[\mathcal{D} v^{k}(s)+\Delta\left(s, z^{k}(s)\right)\right] \varphi^{\prime}(s) d s, & \text { and }\end{cases} \\
& z^{*}(t)= \begin{cases}\mathcal{S}_{\varphi}^{\sigma_{1}, \sigma_{2}}(t, 0)\left[z_{0}-\mathcal{G}\left(z^{*}\right)\right] \\
+\int_{0}^{t}(\varphi(t)-\varphi(s))^{\sigma_{1}-1} \mathcal{T}_{\varphi}^{\sigma_{1}}(t, s)\left[\mathcal{D} v^{*}(s)+\Delta\left(s, z^{*}(s)\right)\right] \varphi^{\prime}(s) d s, \\
\mathcal{S}_{\varphi}^{\sigma_{1}, \sigma_{2}}\left(t, t_{\gamma}\right)\left[z^{*}\left(t_{\gamma}^{-}\right)+\mathcal{I}_{\gamma}\left(z^{*}\left(t_{\gamma}^{-}\right)\right)\right] \\
+\int_{t_{\gamma}}^{t}(\varphi(t)-\varphi(s))^{\sigma_{1}-1} \mathcal{T}_{\varphi}^{\sigma_{1}}(t, s)\left[\mathcal{D} v^{*}(s)+\Delta\left(s, z^{*}(s)\right)\right] \varphi^{\prime}(s) d s, & t \in\left[t_{1}\right], \gamma=0,\end{cases}
\end{aligned}
$$

It follows from the boundedness of $\left\{v^{k}\right\},\left\{v^{*}\right\}$ and Theorem 2, we obtain there exists a constant $\Theta>0$ such that $\left\|z^{k}\right\|_{\infty},\left\|z^{*}\right\|_{\infty} \leq \Theta$.

For every $t \in\left[0, t_{1}\right], \gamma=0$, we get 


$$
\begin{aligned}
\left\|(\varphi(t)-\varphi(0))^{1-\rho}\left[z^{k}(t)-z^{*}(t)\right]\right\| & \leq \mathcal{M}_{1} \hat{\mathcal{K}}_{\mathcal{G}}\left\|(\varphi(t)-\varphi(0))^{1-\rho}\left[z^{k}(t)-z^{*}(t)\right]\right\| \\
& +\mathcal{M}_{2} \hat{\mathcal{R}}_{\Delta}\left(\varphi\left(t_{1}\right)-\varphi(0)\right)^{1-\rho} \int_{0}^{t}(\varphi(t)-\varphi(s))^{\sigma_{1}-1} \\
& \times(\varphi(s)-\varphi(0))^{\rho-1}\left\|(\varphi(s)-\varphi(0))^{1-\rho}\left[z^{k}(s)-z^{*}(s)\right]\right\| \varphi^{\prime}(s) d s \\
& +\mathcal{M}_{2}\left(\varphi\left(t_{1}\right)-\varphi(0)\right)^{1-\rho} \int_{0}^{t}(\varphi(t)-\varphi(s))^{\sigma_{1}-1} \\
& \times\left\|\mathcal{D} v^{k}(s)-\mathcal{D} v^{*}(s)\right\|_{L^{2}\left(\mathcal{J}_{0}, E\right)} \varphi^{\prime}(s) d s \\
& \leq \mathcal{M}_{1} \hat{\mathcal{K}}_{\mathcal{G}}\left\|(\varphi(t)-\varphi(0))^{1-\rho}\left[z^{k}(t)-z^{*}(t)\right]\right\| \\
& +\mathcal{M}_{2} \hat{\mathcal{R}}_{\Delta}\left(\varphi\left(t_{1}\right)-\varphi(0)\right)^{1-\rho} \int_{0}^{t}(\varphi(t)-\varphi(s))^{\sigma_{1}-1} \\
& \times(\varphi(s)-\varphi(0))^{\rho-1}\left\|(\varphi(s)-\varphi(0))^{1-\rho}\left[z^{k}(s)-z^{*}(s)\right]\right\| \varphi^{\prime}(s) d s \\
& +\frac{\mathcal{M}_{2} \mathcal{K}_{*}^{1 / 2}\left(\varphi\left(t_{1}\right)-\varphi(0)\right)^{\sigma_{1}-\rho+(1 / 2)}}{\left(2 \sigma_{1}-1\right)^{1 / 2}}\left\|\mathcal{D} v^{k}-\mathcal{D} v^{*}\right\|_{L^{2}\left(\mathcal{J}_{0}, E\right)} .
\end{aligned}
$$

For every $t \in\left(t_{\gamma}, t_{\gamma+1}\right], \gamma=1,2, \ldots, \mathcal{H}$, we get

$$
\begin{aligned}
\left\|\left(\varphi(t)-\varphi\left(t_{\gamma}\right)\right)^{1-\rho}\left[z^{k}(t)-z^{*}(t)\right]\right\| & \leq \mathcal{M}_{1}\left(1+\mathcal{D}_{\gamma}\right)\left\|\left(\varphi(t)-\varphi\left(t_{\gamma}\right)\right)^{1-\rho}\left[z^{k}\left(t_{\gamma}^{-}\right)-z^{*}\left(t_{\gamma}^{-}\right)\right]\right\| \\
& +\mathcal{M}_{2} \hat{\mathcal{R}}_{\Delta}\left(\varphi\left(t_{\gamma+1}\right)-\varphi\left(t_{\gamma}\right)\right)^{1-\rho} \int_{t_{\gamma}}^{t}(\varphi(t)-\varphi(s))^{\sigma_{1}-1} \\
& \times\left(\varphi(s)-\varphi\left(t_{\gamma}\right)\right)^{\rho-1}\left\|\left(\varphi(s)-\varphi\left(t_{\gamma}\right)\right)^{1-\rho}\left[z^{k}(s)-z^{*}(s)\right]\right\| \varphi^{\prime}(s) d s \\
& +\mathcal{M}_{2}\left(\varphi\left(t_{\gamma+1}\right)-\varphi\left(t_{\gamma}\right)\right)^{1-\rho} \int_{t_{\gamma}}^{t}(\varphi(t)-\varphi(s))^{\sigma_{1}-1} \\
& \times\left\|\mathcal{D} v^{k}(s)-\mathcal{D} v^{*}(s)\right\|_{L^{2}\left(\mathcal{J}_{0}, E\right)} \varphi^{\prime}(s) d s \\
& \leq \mathcal{M}_{1}\left(1+\mathcal{D}_{\gamma}\right)\left\|\left(\varphi(t)-\varphi\left(t_{\gamma}\right)\right)^{1-\rho}\left[z^{k}\left(t_{\gamma}^{-}\right)-z^{*}\left(t_{\gamma}^{-}\right)\right]\right\| \\
& +\mathcal{M}_{2} \hat{\mathcal{R}}_{\Delta}\left(\varphi\left(t_{\gamma+1}\right)-\varphi\left(t_{\gamma}\right)\right)^{1-\rho} \int_{t_{\gamma}}^{t}(\varphi(t)-\varphi(s))^{\sigma_{1}-1} \\
& \times(\varphi(s)-\varphi(0))^{\rho-1}\left\|\left(\varphi(s)-\varphi\left(t_{\gamma}\right)\right)^{1-\rho}\left[z^{k}(s)-z^{*}(s)\right]\right\| \varphi^{\prime}(s) d s \\
& +\frac{\mathcal{M}_{2} \mathcal{K}_{*}^{1 / 2}\left(\varphi\left(t_{\gamma+1}\right)-\varphi\left(t_{\gamma}\right)\right)^{\sigma_{1}-\rho+(1 / 2)}}{\left(2 \sigma_{1}-1\right)^{1 / 2}}\left\|\mathcal{D} v^{k}-\mathcal{D} v^{*}\right\|_{L^{2}\left(\mathcal{J}_{0}, E\right)} .
\end{aligned}
$$

For every $t \in \mathcal{J}_{0}$, we obtain

$$
\begin{aligned}
\left\|z^{k}-z^{*}\right\|_{\mathcal{P C}} & \leq \mathcal{M}_{1}\left(1+\mathcal{D}_{\gamma}\right)\left\|z^{k}-z^{*}\right\|_{\mathcal{P C}}+\mathcal{M}_{1} \hat{\mathcal{K}}_{\mathcal{G}}\left\|z^{k}-z^{*}\right\|_{\mathcal{P C}} \\
& +\mathcal{M}_{2} \hat{\mathcal{R}}_{\Delta} \frac{\Gamma\left(\sigma_{1}\right) \Gamma(\rho)}{\Gamma\left(\rho+\sigma_{1}\right)}(\varphi(b)-\varphi(0))^{\sigma_{1}}\left\|z^{k}-z^{*}\right\|_{\mathcal{P C}} \\
& +\frac{\mathcal{M}_{2} \mathcal{K}_{*}^{1 / 2}(\varphi(b)-\varphi(0))^{\sigma_{1}-\rho+(1 / 2)}}{\left(2 \sigma_{1}-1\right)^{1 / 2}}\left\|\mathcal{D} v^{k}-\mathcal{D} v^{*}\right\|_{L^{2}\left(\mathcal{J}_{0}, E\right)}
\end{aligned}
$$

then there exists a constant $\mathcal{N}^{*}>0$ such that

$$
\left\|z^{k}-z^{*}\right\|_{\mathcal{P C}} \leq \mathcal{N}^{*}\left\|\mathcal{D} v^{k}-\mathcal{D} v^{*}\right\|_{L^{2}\left(\mathcal{J}_{0}, E\right)},
$$

where

$$
\mathcal{N}^{*}=\frac{\mathcal{M}_{2} \mathcal{K}_{*}^{1 / 2}(\varphi(b)-\varphi(0))^{\sigma_{1}-\rho+(1 / 2)}}{\left(2 \sigma_{1}-1\right)^{1 / 2}\left(1-\mathcal{M}_{1}\left(1+\mathcal{D}_{\gamma}\right)-\mathcal{M}_{1} \hat{\mathcal{K}}_{\mathcal{G}}-\mathcal{M}_{2} \hat{\mathcal{R}}_{\Delta} \frac{\Gamma\left(\sigma_{1}\right) \Gamma(\rho)}{\Gamma\left(\rho+\sigma_{1}\right)}(\varphi(b)-\varphi(0))^{\sigma_{1}}\right)},
$$


with $\mathcal{M}_{1}\left(1+\mathcal{D}_{\gamma}\right)+\mathcal{M}_{1} \hat{\mathcal{K}}_{\mathcal{G}}+\mathcal{M}_{2} \hat{\mathcal{R}}_{\Delta} \frac{\Gamma\left(\sigma_{1}\right) \Gamma(\rho)}{\Gamma\left(\rho+\sigma_{1}\right)}(\varphi(b)-\varphi(0))^{\sigma_{1}}<1$ for every $\gamma=1,2, \ldots, \mathcal{H}$

Since $\mathcal{D}$ is strongly continuous, we obtain

$$
\left\|\mathcal{D} v^{k}-\mathcal{D} v^{*}\right\|_{L^{2}\left(\mathcal{J}_{0}, E\right)} \longrightarrow 0 \text { as } k \rightarrow \infty .
$$

Thus, we have

$$
\left\|z^{k}-z^{*}\right\|_{\mathcal{P C}} \longrightarrow 0 \text { as } k \rightarrow \infty,
$$

this yields that $z^{k} \longrightarrow z^{*}$ in $\mathcal{P C}(E)$ as $k \rightarrow \infty$. Since $\mathcal{P C}(E) \subset L^{1}\left(\mathcal{J}_{0}, E\right)$, by using [X9] and Balder's theorem, we obtain

$$
\begin{aligned}
\epsilon & =\lim _{k \rightarrow \infty} \sum_{\gamma=0}^{\mathcal{H}} \int_{t_{\gamma}}^{t_{\gamma+1}} \mathcal{L}\left(t, z^{k}(t), v^{k}(t)\right) d t \\
& \geq \sum_{\gamma=0}^{\mathcal{H}} \int_{t_{\gamma}}^{t_{\gamma+1}} \mathcal{L}\left(t, z^{*}(t), v^{*}(t)\right) d t=\mathcal{J}\left(z^{*}, v^{*}\right) \geq \epsilon, \gamma=0,1, \ldots, \mathcal{H} .
\end{aligned}
$$

Thus $J$ attains its minimum at $v^{*} \in \mathcal{U}_{a d}$.

\section{Example}

Consider the following $\varphi$-Hilfer fractional impulsive differential control system to verify the proposed results:

$$
\left\{\begin{array}{l}
{ }^{H} \mathrm{D}_{t_{\gamma}^{+}}^{\sigma_{1}, \sigma_{2} ; \varphi} z(t, \alpha)=z_{\alpha \alpha}(t, \alpha)+v(t, \alpha)+\frac{t e^{-t} z(t, \alpha)}{18(1+|z(t, \alpha)|)}, t \in(0,1]-\left\{t_{1}\right\}, \alpha \in[0, \pi], \\
I_{t_{1}^{+}}^{\left(1-\sigma_{1}\right)\left(1-\sigma_{2}\right) ; \varphi} z\left(t_{1}^{+}, \alpha\right)=z\left(t_{1}^{-}, \alpha\right)+\frac{1}{100} z\left(t_{1}^{-}, \alpha\right), \alpha \in[0, \pi], \\
I_{0^{+}}^{\left(1-\sigma_{1}\right)\left(1-\sigma_{2}\right) ; \varphi}[z(t, \alpha)]_{t=0}+\frac{1}{15} z(t, \alpha)=z_{0}(\alpha), \\
z(t, 0)=0=z(t, \pi),
\end{array}\right.
$$

with cost functional as

$$
\mathcal{J}\left(z^{v}, v\right)=\sum_{\gamma=0}^{\mathcal{H}}\left[\int_{t_{\gamma}}^{t_{\gamma+1}} \int_{0}^{\pi}\left|z^{v}(t, \alpha)\right|^{2} d \alpha d t+\int_{t_{\gamma}}^{t_{\gamma+1}} \int_{0}^{\pi}|v(t, \alpha)|^{2} d \alpha d t\right]
$$

subject to the problem (13), where $\gamma=0,1, \sigma_{1}=2 / 3, \sigma_{2}=1 / 4$ and $0=t_{0}<t_{1}<t_{2}=b$ with $t_{1}=0.5, b=1$. Let $\varphi(t)=t$ and $E=\mathcal{T}=L^{2}([0, \pi])$. Define an operator $\mathcal{A}: \mathcal{D}(\mathcal{A}) \subseteq$ $E \rightarrow E$ by $\mathcal{A} \psi=\psi^{\prime \prime}$ with

$\mathcal{D}(\mathcal{A})=\left\{\psi \in E: \psi, \psi^{\prime}\right.$ are absolutely continuous and $\left.\psi^{\prime \prime} \in E, \psi(0)=0=\psi(\pi)\right\}$.

$\mathcal{A}$ has a discrete spectrum, the normalized eigenvectors $e_{n}(\alpha)=\sqrt{2 / \pi} \sin (n \alpha)$ corresponding to eigenvalue are $-n^{2}, n \in \mathbb{N}$ and $\mathcal{A}$ generates an analytic semigroup $\{\mathcal{T}(t)\}_{t \geq 0}$ in $E$, which uniformly bounded and defined as

$$
\mathcal{T}(t) \alpha=\sum_{n=1}^{\infty} e^{-n^{2} t}\left\langle\alpha, e_{n}\right\rangle e_{n}, \alpha \in E,
$$

with $\|\mathcal{T}(t)\| \leq e^{-t} \forall t \geq 0$. Thus, we choose $\mathcal{M}=1$ that implies that $\sup _{t \in[0, \infty)}\|\mathcal{T}(t)\|=1$ and [X1] is fulfilled. We obtain $\mathcal{M}_{1}=0.8161$ and $\mathcal{M}_{2}=0.7385$. The admissible controls set

$$
\mathcal{U}_{a d}=\left\{v \in \mathcal{T}:\|v\| \in L^{2}([0,1], \mathcal{T}) \leq 1\right\}
$$


Let $z(t)(\alpha)=z(t, \alpha)$ and the functions $\Delta, \mathcal{I}_{1}$ and $\mathcal{G}$ are defined as

$$
\Delta(t, z)(\alpha)=\frac{t e^{-t} z(t, \alpha)}{18(1+|z(t, \alpha)|)}, \quad \mathcal{I}_{1}=\frac{1}{100} z\left(t_{1}^{-}, \alpha\right), \quad \mathcal{G}(z)(\alpha)=\frac{1}{15} z(t, \alpha) .
$$

We obtain $\mathcal{K}_{\Delta}=\mathcal{R}_{\Delta}=1 / 18, \hat{\mathcal{K}}_{\mathcal{G}}=1 / 15, \mathcal{D}_{1}=1 / 100$ and

1. $\mathcal{O}=\max \left[\mathcal{M}_{1} \hat{\mathcal{K}}_{\mathcal{G}}, \mathcal{M}_{1}\left(1+\mathcal{D}_{1}\right)\right]=\max [0.0544,0.8243]<1$,

2. $\mathcal{M}_{1} \hat{\mathcal{K}}_{\mathcal{G}}+\mathcal{M}_{2} \mathcal{K}_{\Delta} \frac{\Gamma\left(\sigma_{1}\right) \Gamma(\rho)}{\Gamma\left(\rho+\sigma_{1}\right)}(\varphi(b)-\varphi(0))^{\sigma_{1}}=0.1312<1$,

3. $\mathcal{M}_{1}\left(1+\mathcal{D}_{1}\right)+\mathcal{M}_{1} \hat{\mathcal{K}}_{\mathcal{G}}+\mathcal{M}_{2} \hat{\mathcal{R}}_{\Delta} \frac{\Gamma\left(\sigma_{1}\right) \Gamma(\rho)}{\Gamma\left(\rho+\sigma_{1}\right)}(\varphi(b)-\varphi(0))^{\sigma_{1}}=0.9555<1$.

The system (13) can be transformed into (11) with the functional

$$
\mathcal{J}\left(z^{v}, v\right)=\sum_{\gamma=0}^{\mathcal{H}} \int_{t_{\gamma}}^{t_{\gamma+1}}\left[\left\|z^{v}(t)\right\|^{2}+\|v(t)\|_{\mathcal{T}}^{2}\right] d t
$$

All hypotheses of Theorems 3 and 4 are satisfied. Hence, the problem (13) has at least one optimal pair.

\section{Discussion}

The solvability and optimal control results for a class of $\varphi$-Hilfer fractional differential equations with impulses and nonlocal conditions have been investigated. Standard techniques combined with the notion of piecewise continuous mild solutions were used for the main results. Moreover, by using the minimizing sequence concept, we proved the optimal controls for deriving the optimality conditions. At end, we presented an illustrative example to provide the obtained theoretical results. In the forthcoming papers, as new direction, we intend to investigate the relaxation in nonconvex optimal control problems for a new class of $\varphi$-Hilfer fractional stochastic differential equations driven by the Rosenblatt process with non-instantaneous impulses [34,35].

Author Contributions: Conceptualization, A.D.; methodology, M.M.; validation, A.D.; formal analysis, R.D.; investigation, S.G.; writing—original draft preparation, S.G., A.D.; writing—review and editing, A.D., M.M.; supervision, A.D.; project administration, R.D. All authors have read and agreed to the published version of the manuscript.

Funding: This research received no external funding.

Institutional Review Board Statement: Not applicable.

Informed Consent Statement: Not applicable.

Data Availability Statement: Not applicable.

Acknowledgments: We are very thankful to the anonymous reviewers and associate editor for their constructive comments and suggestions which help us to improve the manuscript.

Conflicts of Interest: The authors declare no conflict of interest. The funders had no role in the design of the study; in the collection, analyses, or interpretation of data; in the writing of the manuscript, or in the decision to publish the results.

\section{References}

1. Zhou, Y.; Wang, J.; Zhang, L. Basic Theory of Fractional Differential Equations; World Scientific Publishing Co. Pte. Ltd.: Hackensack, NJ, USA, 2017.

2. Miller, K.S.; Ross, B. An Introduction to the Fractional Calculus and Fractional Differential Equations; A Wiley-Interscience Publication; John Wiley and Sons, Inc.: New York, NY, USA, 1993.

3. Kilbas, A.A.; Srivastava, H.M.; Trujillo, J.J. Theory and applications of fractional differential equations. In North-Holland Mathematics Studies; Elsevier Science B.V.: Amsterdam, The Netherlands, 2006; Volume 204. 
4. Podlubny, I. Fractional differential equations. In Mathematics in Science and Engineering; Academic Press, Inc.: San Diego, CA, USA, 1999; Volume 198.

5. Dhayal, R.; Malik, M.; Abbas, S. Solvability and optimal controls of non-instantaneous impulsive stochastic fractional differential equation of order $q \in(1,2)$. Stochastics 2020, 93, 780-802. [CrossRef]

6. Hilfer, R. Application of Fractional Calculus in Physics; World Scientific: Singapore, 2000.

7. Wang, J.; Zhang, Y. Nonlocal initial value problems for differential equations with Hilfer fractional derivative. Appl. Math. Comput. 2015, 266, 850-859. [CrossRef]

8. Karthikeyan, K.; Debbouche, A.; Torres, D.F.M. Analysis of Hilfer fractional integro-differential equations with almost sectorial operators. Fractal Fract. 2021, 5, 22. [CrossRef]

9. Yang, M.; Wang, Q. Approximate controllability of Hilfer fractional differential inclusions with nonlocal conditions. Math. Methods Appl. Sci. 2017, 40, 1126-1138. [CrossRef]

10. Vijayakumar, V.; Udhayakumar, R. Results on approximate controllability for non-densely defined Hilfer fractional differential system with infinite delay. Chaos Solitons Fractals 2020, 139, 110019. [CrossRef]

11. Debbouche, A.; Antonov, V. Approximate controllability of semilinear Hilfer fractional differential inclusions with impulsive control inclusion conditions in Banach spaces. Chaos Solitons Fractals 2017, 102, 140-148. [CrossRef]

12. Sousa, J.V.C.; Oliveira, E.C. On the $\psi$-Hilfer fractional derivative. Commun. Nonlinear Sci. Numer. Simul. 2018, 60, 72-91. [CrossRef]

13. Sousa, J.V.C.; Kucche, K.D.; Oliveira, E.C. Stability of $\psi$-Hilfer impulsive fractional differential equations. Appl. Math. Lett. 2019, 88, 73-80. [CrossRef]

14. Kucche, K.D.; Kharade, J.P.; Sousa, J.V.C. On the nonlinear impulsive $\psi$-Hilfer fractional differential equations. Math. Model. Anal. 2020, 25, 642-660. [CrossRef]

15. Sousa, J.V.C.; Oliveira, E.C. On the Ulam-Hyers-Rassias stability for nonlinear fractional differential equations using the $\psi$-Hilfer operator. J. Fixed Point Theory Appl. 2018, 20, 96. [CrossRef]

16. Sousa, J.V.C.; Rodrigues, F.G.; Oliveira, E.C. Stability of the fractional Volterra integro-differential equation by means of $\psi$-Hilfer operator. Math. Methods Appl. Sci. 2019, 42, 3033-3043. [CrossRef]

17. Suechoei, A.; Sa Ngiamsunthorn, P. Existence uniqueness and stability of mild solutions for semilinear $\psi$-Caputo fractional evolution equations. Adv. Differ. Equ. 2020, 114. [CrossRef]

18. Malti, A.I.N.; Benchohra, M.; Graef, J.R.; Lazreg, J.E. Impulsive boundary value problems for nonlinear implicit Caputoexponential type fractional differential equations. Electron. J. Qual. Theory Differ. Equ. 2020, 2020, 1-17. [CrossRef]

19. Vadivoo, B.S.; Ramachandran, R.; Cao, J.; Zhang, H.; Li, X. Controllability analysis of nonlinear neutral-type fractional-order differential systems with state delay and impulsive effects. Int. J. Control. Autom. Syst. 2018, 16, 659-669. [CrossRef]

20. Aimene, D.; Baleanu, D.; Seba, D. Controllability of semilinear impulsive Atangana-Baleanu fractional differential equations with delay. Chaos Solitons Fractals 2019, 128, 51-57. [CrossRef]

21. Dhayal, R.; Malik, M.; Abbas, S. Existence, stability and controllability results of stochastic differential equations with noninstantaneous impulses. Int. J. Control 2020, 1-12. [CrossRef]

22. Yu, X.; Debbouche, A.; Wang, J. On the iterative learning control of fractional impulsive evolution equations in Banach spaces. Math. Methods Appl. Sci. 2017, 40, 6061-6069. [CrossRef]

23. Ahmed, H.M.; El-Borai, M.M.; El-Owaidy, H.M.; Ghanem, A.S. Impulsive Hilfer fractional differential equations. Adv. Differ. Equ. 2018, 226. [CrossRef]

24. Kucche, K.D.; Kharade, J.P. Analysis of impulsive $\psi$-Hilfer fractional differential equations. Mediterr. J. Math. 2020, 17, 163. [CrossRef]

25. Wang, J.; Zhou, Y. A class of fractional evolution equations and optimal controls. Nonlinear Anal. Real World Appl. 2011, 12, 262-272. [CrossRef]

26. Dhayal, R.; Malik, M.; Abbas, S.; Debbouche, A. Optimal controls for second-order stochastic differential equations driven by mixed-fractional Brownian motion with impulses. Math. Methods Appl. Sci. 2020, 43, 4107-4124. [CrossRef]

27. Debbouche, A.; Nieto, J.J. Sobolev type fractional abstract evolution equations with nonlocal conditions and optimal multi-controls. Appl. Math. Comput. 2014, 245, 74-85. [CrossRef]

28. Liu, S.; Wang, J. Optimal controls of systems governed by semilinear fractional differential equations with not instantaneous impulses. J. Optim. Theory Appl. 2017, 174, 455-473. [CrossRef]

29. Balasubramaniam, P.; Tamilalagan, P. The solvability and optimal controls for impulsive fractional stochastic integro-differential equations via resolvent operators. J. Optim. Theory Appl. 2017, 174, 139-155. [CrossRef]

30. Yan, Z.; Jia, X. Optimal controls of fractional impulsive partial neutral stochastic integro-differential syste Infin. Delay Hilbert Spaces. Int. J. Control. Autom. Syst. 2017, 15, 1051-1068. [CrossRef]

31. Harrat, A.; Nieto, J.J.; Debbouche, A. Solvability and optimal controls of impulsive Hilfer fractional delay evolution inclusions with Clarke subdifferential. J. Comput. Appl. Math. 2018, 344, 725-737. [CrossRef]

32. Zhou, Y.; Jiao, F. Existence of mild solutions for fractional neutral evolution equations. Comput. Math. Appl. 2010, 59, 1063-1077. [CrossRef]

33. Sakthivel, R.; Revathi, P.; Ren, Y. Existence of solutions for nonlinear fractional stochastic differential equations. Nonlinear Anal. Theory Methods Appl. 2013, 81, 70-86. [CrossRef] 
34. Debbouche, A.; Nieto, J.J.; Torres, D.F.M. Optimal solutions to relaxation in multiple control problems of Sobolev type with nonlocal nonlinear fractional differential equations. J. Optim. Theory Appl. 2017, 174, 7-31. [CrossRef]

35. Debbouche, A.; Nieto, J.J. Relaxation in controlled systems described by fractional integro-differential equations with nonlocal control conditions. Electron. J. Differ. Equ. 2015, 2015, 1-18. 